\title{
The effect of melodic and temporal contour on recognition memory for pitch change
}

\author{
CAROLINE B. MONAHAN \\ Central Institute for the Deaf, St. Louis, Missouri \\ and \\ ROGER A. KENDALL and EDWARD C. CARTERETTE \\ University of Califormia, Los Angeles, Califormia
}

\begin{abstract}
This study was designed to explore the kinds of temporal patterning that foster pitch-difference discrimination. Musicians and nonmusicians rated the similarity of pairs of 9-note melodies that could differ in the pitch chroma of a single note at any of five serial positions. In a complete factorial design, there were 84 standard melodies (4 pitch patterns $\times 21$ rhythms), each of which was paired with 10 octave-raised comparisons; 5 comparisons were identical to the standard in chroma and 5 had a single changed chroma. A literature review suggested that temporal accent occurs for tones initiating a lengthened temporal interval and for tones initiating a group of three or more intervals; pitch-level accent is a product of pitch skips on the order of 4 semitones or of the change of direction of the pitch contour. In this study there were three classes of temporal patterns. Rhythmically consonant patterns had temporal accenting that was always metrically in phase with pitch-level accenting and promoted the best performance. Rhythmically out-of-phase consonant patterns had temporal accenting and pitch-level accenting that occurred regularly at the same metrical rate, but the two were never in phase. Rhythmically dissonant patterns had temporal accenting and pitch-level accenting at different metrical rates. Patterns in the latter two classes sound syncopated, and they generally resulted in poorer pitch-discrimination performance. Musicians performed better than nonmusicians on all patterns; however, an account of performance in terms of "rhythmic nonconsonance" generated by the above three categories predicted $63 \%$ and $42 \%$ of the variance in musicians' and nonmusicians' performance, respectively. Performance at all serial positions was generally best for tones initiating long sound-filled intervals and was also better at a particular serial position when pitch-level accenting took the form of a pitch contour inflection instead of a unidirectional pitch skip. There was some evidence that rhythmic consonance early in a pattern improved musicians' performance at a later serial position.
\end{abstract}

According to Cooper and Meyer (1960), accenting is the basis for grouping in melodies. We assume that melodies whose pitches may be easily grouped constitute "'good Gestalts" that are more easily coded and remembered. Accent is a perceptual phenomenon that is usually but not necessarily correlated with cues that may occur in each of several physical dimensions. Monahan and Carterette (1985) distinguished five major sources of cues for accent in monophonic melodies: (1) temporal patterning, (2) pitch-pattern shape (pitch contour and pitch interval sizes), (3) dynamic patterning, (4) the tonal system to which the set of pitches belong, and (5) timbral patterning. In our discussion we will refer to physical cues as accenting and to perceptual phenomena as accent.

Support for statistical analysis of the data was provided by a grant to the Central Institute for the Deaf by the Air Force Office for Scientific Research. The authors thank Mark Pitt for writing the computer program that collected listeners' responses, Andrew DeGruccio for running the experiment, and Ted Meyer for helping prepare data for analysis. We also thank Marios Fourakis, Janet Weisenberger, and Ira J. Hirsh for their comments on earlier drafts of portions of the present paper. Address correspondence to Caroline B. Monahan, Central Institute for the Deaf, 818 S. Euclid Ave., St. Louis, MO 63110.
Below, we consider accent and ease of grouping from the standpoint of (1) the organization of temporal intervals alone and (2) pitch pattern shape alone. The advantage of choosing these two cue sources is that they may be varied in ways that are physically independent of each other; therefore, perceptual dependencies between these dimensions are not confounded with stimulus dependencies.

We also consider three possible relationships between accenting from these two sources when cues from each source occur regularly with fixed periodicity: rhythmic consonance, dissonance, and out-of-phase consonance (see Monahan \& Carterette, 1985; Yeston, 1976). We hypothesized that patterns with rhythmically consonant accenting will promote easier grouping and therefore better coding and memory for the pitches of the pattern.

We examine in detail a previous experiment by Jones, Boltz, and Kidd (1982) that suggested discrimination of pitch differences between two musical patterns was a product of rhythmic patterning and whether or not a higher-order pitch rule was instantiated at a particular serial position. Jones et al.'s manipulation of rhythmic context raised two major questions (1) What sorts of tem- 
poral events are associated with better pitch-difference detection (e.g., relatively longer temporal intervals that are sound-filled or not, or temporal intervals that follow silences)? and (2) Does temporal organization in one part of a melodic pattern affect pitch-difference recognition in another part of the pattern?

Based on our hypothesis of better pitch coding in rhythmically consonant patterns and questions raised by Jones et al. (1982), we'investigate here some of the combined effects of pitch pattern shape and temporal patterning on a listener's ability to discriminate patterns that differ in a single pitch at a particular serial position.

\section{BACKGROUND}

\section{Temporal Accenting}

Several recent papers have been explicitly devoted to the description of time in a musical context (e.g., Handel \& Lawson, 1983; Handel \& Oshinsky, 1981; LonguetHiggins \& Lee, 1982; Monahan \& Carterette, 1985; Povel, 1981, 1984; Povel \& Essens, 1985; Steedman, 1977; Yeston, 1976). All of these authors assume that time in music is psychologically arranged in a hierarchy as a rate within a rate. That is, musical time is a periodic pulse or temporal grain, a string of equal intervals (some of which are marked by note onsets and some of which are not) upon which the listener superimposes a slower periodic accent rate, metric, clock, or beat (all of these terms are equivalent).

The strongest natural temporal accent tends to appear on tones initiating relatively lengthened intervals (as measured from the attack point of one tone to the attack of the next, denoted IOI for interonset interval); this phenomenon, usually measured by subjects' reports or by tapping, occurs for both equitone sequences in which temporal intervals are marked with tones of the same length, intensity, and frequency (Povel \& Essens, 1985; Povel \& Okkerman, 1981), and monotone sequences in which sound-filling is a fixed proportion of all intervals (Vos, 1977; Woodrow, 1909).

Starting with an isochronous equitone sequence, Povel and Okkerman (1981) gradually increased the length of every third interval. At first, listeners reported hearing triples of intervals with an accent on the tone ending the lengthened interval; however, as the ratio of the length of the longer IOI to either shorter IOI approached 2:1, listeners reported the strongest temporal accent on the tone beginning the longer interval, while continuing to hear a much weaker accent on the tone ending the lengthened interval.

\section{Grouping of Temporal Intervals}

For continuously repeating patterns, Vos (1977) demonstrated that the perception of common poetic rhythms can be induced in listeners simply by manipulating the two components of the IOI: the sound-filled tone-duration interval (TDI) and the silent interval (SI) from the offset of the tone to the onset of the following tone. His most general finding was that the longer the IOI, the more likely it was to sound accented and to end a rhythmic group. This finding fits with the Gestalt principles and with previous work by Garner (1974), who termed a similar finding "the gap principle." However, Vos found he could create the perception of trochaic rhythmic grouping (accented tone followed by unaccented tone) by having IOIs that were equal in length, with every other IOI having a longer tone duration; the interval with the longer SI tended to end the group and the one with the longer TDI sounded accented. If temporal intervals (IOIs) are thought of as having two parts, one sound-filled and the other silent, the silent portion is the dominant aspect in grouping, and the sound-filled portion in accenting. In most music, when there is more than one length of IOI, temporal intervals either tend to fit the equitone pattern (as with bongo drums or any instrument played in a relatively staccato manner) or they are expected to be filled with roughly the same proportion of sound (any instrument played in relatively legato fashion); in either of these cases, longer IOIs tend to form end-accented groups. Also, as noted by Povel and Okkerman (1981), a weaker natural temporal accent apparently occurs on the tone that ends the lengthened temporal interval, if that tone is heard as beginning a group of three or more temporal intervals. However, Vos's results suggest that, whatever note values appear in a score, the performer has enormous latitude in creating groupings by varying the proportion of soundfilling within the indicated IOI.

\section{The Simplicity of Coding Temporal Patterns}

Prescriptions for determining metrical simplicity have been proposed by Yeston (1976), Monahan (1984), and Povel and Essens (1985). In several elegantly designed experiments, Povel and Essens studied listeners' ability to code and reproduce (by tapping a key) the independent permutations of various combinations of temporal intervals. The reason for exploring these pattern sets is that they tend to evoke different accent clocks on the part of the listener, and yet they comprise the same number and kinds of intervals.

Povel and Essens (1985) assumed that perceivers attempt to generate an internal clock or metric when listening to temporal sequences. They hypothesized that the distribution of accenting and hence the perception of "naturally accented events" (discussed above) determines (1) whether an internal clock can be generated and (2) which internal clock is best, or most likely to be induced. They developed an algorithm for predicting perceived metricality, or the strength of induction of a best clock in listeners by recycling equitone sequences. The best clock is hierarchical, because it has two levels: one defines the unit, or metric, and the other determines the subdivision of the unit, just as time on a watch has minutes and seconds. Best clocks may also differ in the serial position in the pattern at which they start.

The three major rules of the algorithm, in descending order of importance, are: (1) The best clock or metric 
must divide the total period of the pattern integrally. (2) The metric should avoid coincidence with intervals that have no event onsets. (3) The metric should avoid coincidence with intervals that have naturally unaccented events. Monahan's (1984) prescription for metrical simplicity in nonrepeating temporal patterns included the first two rules above. Although Povel and Essens (1985) stated the major positive requirement for best clock inductionthat is, that the listener's internal metric should coincide with the onset of naturally accented events-their algorithm considers only the negative evidence of Rules 2 and 3 above in deciding the likelihood of best clock induction (D. J. Povel, personal communication, April 1986). Povel and Essens demonstrated that the number of times Rules 2 and 3 are violated is directly related to the number of times a listener must hear a pattern before he/she feels competent to tap it out, and also to the temporal variability of the intervals that are tapped out.

Let us examine how nonrepeating patterns such as those employed in the present experiment might allow listeners to generate an internal clock. Figure 1 shows a few of these patterns on a time line of 12 equal intervals, where every interval represents $300 \mathrm{msec}$ and note onsets are marked with a slash. We denote the shorter IOI $(300 \mathrm{msec})$ as 1 and the longer $(600 \mathrm{msec})$ as 2 . We will refer to sequences of IOIs as IOI patterns or subpatterns. These patterns can also be described as three consecutive bars, each of which contains 1 quarter note and 2 eighth notes in one of the following rhythmic subpatterns: 211 (dactyl), 112 (anapest), or 121 (amphibrach). We do not mean to imply that listeners will perceive these rhythmic groups when they are concatenated with one anotherquite the contrary.

If the listener does not generate an internal clock, rhythmic grouping should be determined by the contiguity of note onsets: where there is no note onset, the listener is likely to end a rhythmic group. However, if he/she imposes an internal clock, then different groupings can be created. Theoretically, all of these patterns can be fit by internal clocks that are 2, 3, 4, or 6 intervals in length, because all of these clocks may integrally divide the total period of 12 intervals. In practice, most listeners will try to impose the shorter 2- or 3-interval clocks that in most of Western music are nested within longer clocks of 4 or 6 intervals as $2(2)$ or $2(3)$, respectively. However, if the listener could succeed in generating an internal clock every 4 intervals, starting with the first interval, then he/she could hear dactyl, anapest, or amphibrach bars concatenated with each other.

For each pattern, Figure 1 shows the intervals that will receive the most natural accent and also the best clock that listeners could impose by Povel and Essens's (1985) criteria. For Patterns A through D (combinations of 211 with 112 bars), the best clock ticks every 2 (and also every 4) intervals; the best clocks for Patterns $C$ and $D$ and all subsequent patterns (except I) violate Povel and Essens's Rule 3 by coinciding with naturally unaccented events.
The best clock for Patterns E and F (combinations of 211 or 112 with a 121 center) ticks every 4 intervals. However, note that the beginnings and endings of these patterns favor a 2 -interval clock. A 2-interval clock would tick at the onsets of intervals $1,3,5,7,9$, and 11 , but since there is no note onset at interval 7 , such a clock would violate Povel and Essens's Rule 2. Most listeners will hear syncopation in these patterns, since the initial 2-interval metric is displaced in the 121 center.

Temporal accenting in patterns $\mathrm{G}$ and $\mathrm{H}$ (combinations starting and ending with 121 and having 112 or 211 centers) tend to favor the weak induction of a 3-interval best clock. Note that these patterns can also be weakly fit by a 4-interval internal clock. Such patterns are somewhat like ambiguous figures and show the potential for syncopation when combined with nontemporal sources of accenting, because they can support internal clocks in two different metrics. In practice, only one clock or the other is induced by temporal patterning alone (Povel \& Essens, 1985, Experiment 3).

The all-121 pattern (I) most strongly induces a 2- or 4-interval clock that starts on the second interval, so the clock is not commensurate with either end of the pattern. It is debatable whether such a clock should be considered a best clock, because in our nonrepeating patterns, 1interval notes that do not fit within the clock are left dangling at both ends of the pattern. However, since the first note of this pattern may be heard as an upbeat and since the last may be considered to be longer than one interval because it is followed by silence, there is little evidence contrary to the induction of this clock. A 4-interval clock starting on the first interval is also possible, but is extremely unlikely to be induced by temporal accenting, because it breaks Rule 3 by coinciding with unaccented events on three occasions.

From the standpoint of temporal patterning alone, it would seem that combinations involving 121 with 211 or 112 bars would take longer to code and would be less accurately reproduced by finger tapping. Whether the presumed difficulty in temporal coding would affect discrimination of pitch changes when different melodic patterns are combined with these rhythms was open to empirical investigation. We also note that the best-clock algorithm considers only the arrangement of IOIs, and not the proportion of the IOI that is sound-filled, as a basis for difficulty of temporal coding. We intended to investigate whether differential sound-filling of the longer IOIs of a sequence would make a difference in listeners' discrimination of pitch changes.

\section{Pitch-Level Accent}

We distinguish pitch level from tonal sources of accenting, although the two are intimately related. Pitch level is a function of the "shape" or contour of a melody-its succession of ups and downs and the relative size of the pitch distance (in semitones) between notes.

Pitch structure in Western music has traditionally been understood in terms of tonality or musical key. The tonic 


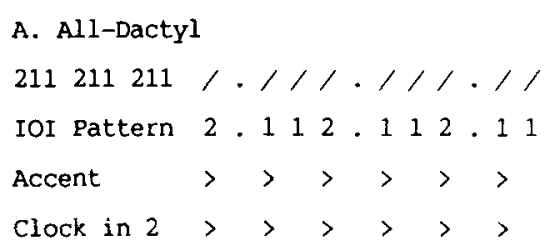

C. Dactyl-Frame Anapest-Center

$211112211 / . / / / /$ / / / /

IOI Pattern 2.1111112 .0 .2 .11

Accent

Clock in $2>>>>>>$

E. Dactyl-Frame Amphibrach-Center

$211121211 / . / / / . / / / /$

IOI Pattern 2.11112 .12 .12 .11

Accent

Clock in $4>$

G. Amphibrach-Frame Dactyl-Center

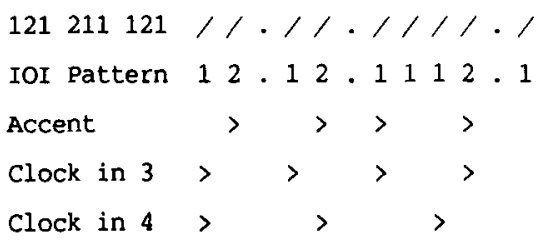

I. All-Amphibrach

$121121121 / / . / / \% / / 1 / 1$

IOI Pattern 12.1112 .112 .1

Accent $>>>>>$

Clock in $2>>>>>$

Clock in $4>>>$
B. All-Anapest

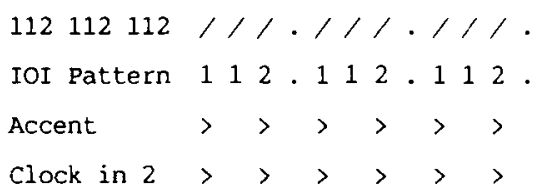

D. Anapest-Frame Dactyl-Center

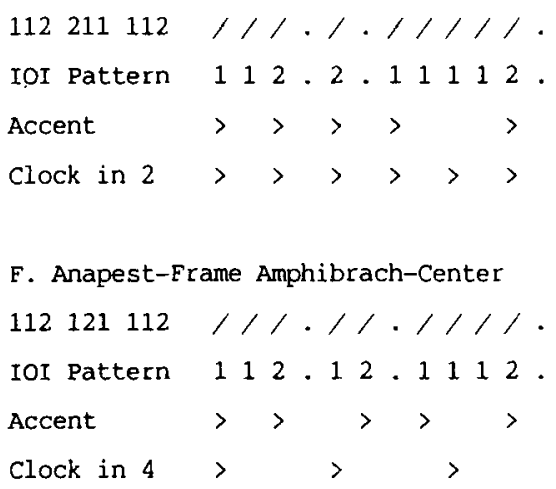

H. Amphibrach-Frame Anapest-Center

$121112121 / / . / / /$ / / / /

IOI Pattern 122.111122 .12 .1

Accent $>>>>$

Clock in $3>>>>$

Clock in $4>>>$

\begin{abstract}
Figure 1. Combinations of dactylic (211), anapestic (112), and amphibrach (121) bars are shown on a time line of 12 equal intervals. Also shown are the position of natural temporal accent and the position of the most strongly induced (best) internal clock(s) that a listener might generate in order to hear a regular beat or metric. Internal clocks will be most strongly evoked if they coincide with intervals that receive natural temporal accent and avoid coincidence with intervals that have no note onset. Patterus A-D strongly evoke 2- or 4-interval clocks starting on the first interval. Patterns $E$ and $F$ weakly evoke a 4 -interval clock. Patterns $G$ and $H$ are somewhat ambiguous; they favor the evocation of a 3-interval clock, but a 4 -interval clock is almost as strongly evoked. Pattern I strongly evokes 2 - and 4-interval clocks that start on the second interval; a much weaker 4-interval clock starting on the first interval theoretically can be evoked, but in practice this is not likely.
\end{abstract}

or abstract tonal center functions as a reference for the pitch-interval relations (and chords) of the whole pattern. Within an octave, each musical key is associated with a hierarchy of 12 pitches that differ in their perceptual salience and stability. These hierarchies seem to be highly influenced by musical context-especially, for Western listeners, the major and minor modes-and by listeners' musical training (Krumhansl \& Kessler, 1982; see also
Dowling \& Harwood, 1986, chap. 4, for a recent review of literature on the perception of scales and tonality). As noted by these researchers, the tonic usually occurs near the beginning and at the end of a musical piece, and often at major phrase boundaries.

Pitch pattern shape, independent of tonality, is thought to generate pitch-level accent. In order to control listeners' rhythmic anticipation, Thomassen (1982) created metri- 
cal contexts by increasing the intensity $(+4 \mathrm{~dB})$ of every third or fourth tone of an isochronous monotone pattern; in these patterns he embedded sequences that varied in frequency. He found that every change in frequency between 2 notes tended to form a pitch-level accent on the second note. Successive changes of pitch in opposite directions tended to accentuate the middle note, for example, C4 E4 C4 accent underlined). Two successive changes of pitch in the same direction gave the impression of accent equally on the second and third notes, for example, C4 E4 $\mathrm{G} \$ 4$. The effect of relative magnitude (interval size) was less pronounced than the effect of contour inflection. In 3- and 4-tone motifs, when the size of successive intervals clearly diverged and especially when the intervals were in the same direction, then the largest was the signal for accenting (e.g., C4, C $\$ 4, G \$ 4)$.

Pitch intervals of more than 5 semitones from one note to the next are exceedingly rare, constituting less than $10 \%$ of all intervals for numerous cultures investigated (Dowling, 1968). Dowling and Harwood (1986) stated that this is a musical universal and is probably based on the physiological structure of the auditory system. If successive notes are 4 or more semitones apart and are presented at a fast rate ( 8 to 10 notes per second), then the single line of notes seems to split into two streams. One stream or the other can be attended to, but not both at once; furthermore, the order of tones in a sequence is difficult to report. This phenomenon has been termed melodic fission (Dowling, 1968,1973 ) or primary auditory stream segregation (Bregman \& Campbell, 1971).

By varying both the presentation rate and the pitch interval, van Noorden (1975) mapped the boundaries of melodic fission on the one hand, and temporal coherence on the other. Melodic patterns that fall between these two boundaries can be heard either as coherent or as split into streams, depending on the listener's attentional strategy. The splitting of tones into particular auditory streams depends not only on the proximity of their frequencies and rates of presentation, but also on whether the sequences are unidirectional or bidirectional. Patterns such as C4 G4 D5 (unidirectional) and C4 G4 C4 (bidirectional) were presented at rates of 7 to 20 tones per second with a 1 -sec pause between patterns. Streaming was far more likely and temporal coherence far less likely to be heard at slower rates for the bidirectional pattern; unidirectional patterns have the Gestalt character of "good continuation," but bidirectional patterns present the listener with a conflict between temporal and frequency proximity. In the latter case, frequency contiguity between the first and third tones totally overrides the temporal contiguity of the first and second tones; temporal coherence is completely lost and the second tone "comes loose" from the other two and forms its own stream.

We note that the very pitch pattern structures that Thomassen (1982) described as providing pitch-level accent at a moderate tempo (slightly less than 5 tones per second) are those that give rise to streaming at faster tempos. If we combine Thomassen's results with those on streaming, then it would seem that points of pitch-level accent are (1) highly noticeable and (2) high likely to be heard as instantiating a different grouping. Tones at the beginnings of musical phrases are very often preceded by relatively large intervals (greater than 4 semitones), followed by intervals that change the direction of the preceding interval, or both. In Western music, any lack of temporal coherence caused by a large pitch interval preceding the pitch at the beginning of a phrase is presumably partly overridden by the typical stability and memorability of that pitch in the system of tonality that has been evoked by the preceding pitches of the pattern.

\section{The Simplicity of Pitch Patterning}

Previously, many theorists and researchers have concentrated on the organization of pitches or pitch intervals by rule, and not on pitch-level accent as a basis for determining melodic grouping or simplicity (Collard \& Povel, 1982; Deutsch \& Feroe, 1981; Dowling, 1971; Jones, 1974, 1976, 1978, 1981; Restle, 1970; Simon \& Sumner, 1968). The research of these workers has been directed at two major hypotheses: (1) certain pitch rules or rule combinations will be easier to learn or recognize than others; (2) the shorter the length of the rule code, the easier the pattern will be to learn. We will address each in turn.

Although systems of notation differ depending on the tasks involved, most of the above researchers have coding systems that allow for repetition, transposition (movement of a pattern or pitch up or down), inversion ( $180^{\circ}$ rotation of a pitch pattern, so that upward motion becomes downward and vice versa), and retrograde $\left(180^{\circ}\right.$ rotation in time, so that the last pitch is first and the first is last). Dowling $(1971,1972)$ and Dowling and Fujitani (1971) showed that listeners could easily recognize repeated or transposed patterns, and that these were better recognized than inverted or retrograde patterns. We note that the above studies involved only isochronous patterns.

The second hypothesis above is usually taken to mean that hierarchically symmetrical patterns of pitches that can be described by nested or recursive rules will be easier to learn than patterns that can be described only by the concatenation of several rules in linear fashion. Dowling and Harwood (1986) noted that most melodies are not perfectly symmetrical and therefore cannot be described by a homogeneous tree structure. Jones (1981) and Boltz and Jones (1986) pointed out that linear rule systems can allow for limited amounts of rule recursion. In an experiment in which pitch and temporal patterning were varied independently, Boltz and Jones showed that linear pitch patterns were transcribed by musicians as well as were those following recursive rules; furthermore, pitch patterns that could be described by either of these rule organizations were easier to transcribe than were no-rule patterns.

Jones and her coworkers have devoted enormous effort to relating pitch rules to temporal or durational sources of accenting (Boltz \& Jones, 1986; Jones et al., 
1982; Jones, Maser, \& Kidd, 1978). Jones et al. (1978) showed that whatever pitch rule system was chosen, it should permit only small changes in pitch over short time periods (lower-level rule span) and large pitch changes over long time periods (higher-level rule span). Patterns that were not organized in this fashion tended to form pitch streams, as described above, and their pitches were not remembered well. In the absence of conflicting temporal grouping and accenting, listeners' perception of a pitch grouping can be expected to jibe with that described by a lower-level pitch rule if the pitch intervals within the span of a lower-level rule are absolutely small (4 semitones or less) and are, on the average, smaller than the pitch intervals between the end of one lower-level rule and the beginning of the next. Jones et al. (1982) and Boltz and Jones (1986) employed only successive units in the diatonic major scale in their lower-level pitch rules. For instance, a pattern such as C4 D4 E4 G4 A5 B5 will be grouped into two parts not only because a repeated lowerlevel rule of +1 diatonic scale steps (or +2 semitone steps) relates $\mathrm{G} 4, \mathrm{~A} 5$, and $\mathrm{B} 5$ just as it does $\mathrm{C} 4, \mathrm{D} 4$, and $\mathrm{E} 4$, but also because the largest pitch skip in the pattern $(+3$ semitones) precedes G4.

It seems to us that the writing of rule systems or musical grammars to describe a listener's knowledge of musical structure is premature until we better understand what may constitute a lower-order rule versus a higher-order rule. In other words, does the pitch-grouping rule written on paper usually match the listener's perception? Furthermore, we should expect to have to write different rules for nonmusicians and musicians (and also for members of other cultures) to reflect their differing knowledge regarding such organizational structures as tonal and metrical hierarchies. In the case of a lower-level rule, it seems we should be searching for the psychophysical laws that constrain and define what musicians usually call a motif: "the briefest intelligible and self-existent melodic or rhythmic unit, consisting of 2 notes or more " (Scholes, 1964, p. 382). Shortterm memory sets an upper limit of about 7 on the number of perceptually unorganized notes; the ability to resolve patterns into separate pitches starts to disappear at rates above 10 notes per second, and patterns tend to lose coherence at rates slower than 1 note per second. Therefore, a motif could be as short as 300 to $400 \mathrm{msec}$ or as long as about $7 \mathrm{sec}$.

\section{Pitch-Level Accenting Combined With Temporal Accenting}

As noted, the beginning of a melodic phrase is often preceded by an unusual pitch skip or marked by a pitch contour inflection; the way the periodicity of this pitchlevel accenting matches with the periodicity of the temporal accenting should determine the listener's ability to code and remember the pitches of a pattern. Monahan and Carterette (1985) suggested this hypothesis, based on Yeston's (1976) structural theory of metrical consonance, dissonance, and out-of-phase consonance. Our formulation is intended for monophonic melodies and its generality may be limited to extremely simple diatonic-scale- step patterns such as those we employed as stimuli in the present experiment. We incorporate Yeston's classifications of metrical structure and Povel and Essens's (1985) notions about the placement of natural accent and their best-clock model for the codability of temporal patterns with Thomassen's (1982) evidence of pitch-level accent. We suggest that three primary components are necessary to classify and index the difficulty of coding pitch patterns with a recurring event period. First, we must take into account the metrical distance between temporal points of accenting (measured in equal intervals); second, the metrical length of a second clock based on pitch-level accenting must be superimposed on the temporal clock; third, the phase relationship between the two clocks will determine the difficulty of pitch-pattern coding. In other words, it is the regularity of pitch events in time, and not just the regular order of pitch event occurrence, that determines the listener's ability to remember the pitches of a pattern. Recently, Boltz and Jones (1986) proposed a model for the simplicity of coding pitch patterns that incorporates as its main feature the ratio of the rates of temporal and pitch-level accenting. Their model is incomplete because it does not consider the difference between temporal accenting and temporal grouping (contiguity of note onsets), nor does it consider fully that the clocks may have different rates or that there are patterns in which temporal and pitch-level accenting are always out of phase.

\section{Rhythmic Consonance}

The three examples of Figure 2 employ the same pitch pattern (one of those we used in the present experiment). The pattern has pitch-level accenting every 4 intervals and every third note onset, beginning with the first note. The examples incorporate the all-anapest, dactyl-frame anapest center, and anapest-frame amphibrach-center rhythms from Figure 1. The spacing in intervals between accenting points gives an indicator of the metrical base of the clock for each of the two cue sources. The phase relationship between the two accent sources is found by looking at the IOI pattern (in eighth-note pulses) for the two sources combined. For the first two patterns, the pitchlevel accenting is perfectly in phase with either the temporal accenting for tones that initiate a long IOI or the temporal accenting for the tone beginning a grouping of three or more temporal intervals. These are rhythmically consonant temporal patterns because both clocks have the same prime-number base (namely 2) and the clocks are in phase-every two 2-interval ticks of the temporal accent clock are perfectly aligned with and nested within a 4-interval tick of the pitch-level accent clock. Pitchdifference detection between two patterns should be easiest for any combination of 211 and 112 frames and center rhythms, because they evoke a clock that is in consonant motion with the pitch-level accent clock.

Pitch-level accenting groups pitches by their contiguity in hertz; thus, the pitch metric in Figure 2 indicates groups that are contiguous in pitch. Grouping by temporal contiguity of note onsets tends to make the long intervals in these patterns end groups of tones; we have also indicated 


\begin{tabular}{|c|c|c|c|c|c|c|c|c|c|c|}
\hline A. Pitch Onsets in Time & $\mathrm{C} 4$ & I & D4 & E4 G4 & . & A5 B5 & $5 \mathrm{C} 4$ & . & D4 & $\mathrm{E} 4$ \\
\hline Pitch-Level Accenting & $>$ & & & $>$ & & & $>$ & & & \\
\hline Pitch Metric & $(4$ & & & )$(4$ & & & )$(4$ & & & 1 \\
\hline Anapest Frame \& Center & $(1$ & 1 & 2 & ).(1$ & 1 & 2 & .) $(1$ & 1 & 2 & )$. \\
\hline Temporal Best Clock & $>$ & & $>$ & $>$ & & $>$ & $>$ & & $>$ & \\
\hline IOI Pattern of Accents & 2 & . & 2 & - 2 & - & 2. & 2 & . & 2 & . \\
\hline B. Pitch Onsets in Time & $\mathrm{C} 4$ & D4 & E4 & G4 & - & A5 B5 & $5 \mathrm{C4}$ & $\mathrm{D} 4$ & E4 & - \\
\hline Pitch-Level Accenting & $>$ & & & $>$ & & & $>$ & & & \\
\hline Pitch Metric & $(4$ & & & )$(4$ & & & 114 & & & 1 \\
\hline Dactyl Frame-Anapest Center & $(2$ & & $(1$ & 11 & 1 & 2 & ).(2$ & )($. & $(1$ & 1 \\
\hline Temporal Best Clock & $>$ & & $>$ & $>$ & & $>$ & $>$ & & $>$ & \\
\hline IOI Pattern of Accents & 2 & - & 2 & - 2 & . & 2. & . 2 & . & 2 & \\
\hline c. Pitch Onsets in Time & $\mathrm{C} 4$ & D4 & E4 & - $\mathrm{G} 4$ & A5 & . $\mathrm{B} 5$ & $5 \mathrm{C4}$ & $\mathrm{D} 4$ & E4 & . \\
\hline Pitch-Level Accenting & $>$ & & & $>$ & & & $>$ & & & \\
\hline Pitch Metric & $(4$ & & & )$(4$ & & & )$(4$ & & & ) \\
\hline Anapest Frame-Amph. Center & $(1$ & 1 & 2 & ).(1$ & 2 & ).(1$ & 11 & 1 & 2 & )$. \\
\hline Temporal Accenting & $>$ & & $>$ & & $>$ & $>$ & $>$ & & $>$ & \\
\hline Temporal Best Clock (Weak) & $>$ & & & $>$ & & & $>$ & & & \\
\hline IOI Pattern of Accents & 2 & & 2 & 1 & 2 & . 1 & 12 & - & 2 & \\
\hline
\end{tabular}

Figure 2. Rhythmically consonant patterns. In Patterns $A$ and B, pitch-level accenting is in consonant motion with the temporal best clock. In Pattern C, while pitchlevel accenting is in phase with the best clock, the best clock is weak and there is a temporary break in consonant motion in the middle of the pattern.

groups that are contiguous in time by parentheses in the IOI pattern for each stimulus. Pitch-difference detection will probably be easiest for patterns that have anapest frames (such as Pattern A in Figure 2), because grouping by temporal contiguity and grouping by pitch contiguity are perfectly in phase at the start of these patterns and listeners can most clearly hear the simple +1 or -1 diatonic-scale-step pitch rule that relates tones of the subpatterns.

The third pattern should be a bit more difficult for listeners, because an internal temporal clock (in base of 2 or 4 ) is less strongly evoked and the IOI pattern for the onsets of accents from each of the two sources reflects this. All patterns with 112 and 211 frames are in basically consonant motion with pitch-level accenting; however, those that have a 121 center are syncopated, or indicate the temporary presence of temporal accenting in a metric that conflicts with the best-clock accent metric.

\section{Out-of-Phase Consonance}

The first example of Figure 3 incorporates the allamphibrach pattern from Figure 1 and the same pitch pat- tern as in Figure 2. Although the clocks have the same prime metrical base (2) and one is perfectly temporally predictable from the other, they are permanently out of phase. In the example given, we may think of the pitchlevel accenting clock as starting 1 pulse ahead of the temporal accenting clock, and the twain never meet. Listeners should have more difficulty in hearing a pitch rule whose onset does not regularly coincide with temporal accenting. The IOI pattern of accent attacks from the two sources is an index of this difficulty and shows where they fail to coincide (where the regular beat every 2 eighth notes fails to occur). The pitch-level accenting periodicity implies a chain or hierarchy of accents starting on the first note, whereas the temporal accenting periodicity implies a chain or hierarchy of accents starting on the second note; since the two never jibe, the pitches of the pattern will not be as easy to group or remember. We hypothesized that pitch-difference discrimination in patterns that have out-of-phase consonant accenting would be quite difficult.

\section{Rhythmic Dissonance}

The second example of Figure 3 incorporates the amphibrach-frame anapest-center pattern from Figure 1 


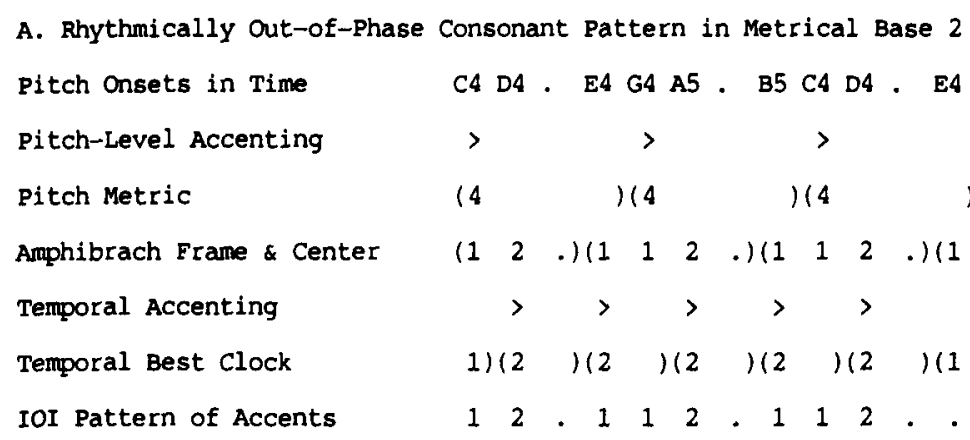

B. Rhythmically Dissonant Pattern ( $3 \times 4$ Polyrhythm)

\begin{tabular}{|c|c|c|c|c|c|c|c|c|c|c|c|c|c|c|c|c|}
\hline Pitch Onsets in Time & & & $\mathrm{C4}$ & $D 4$ & . E & E4 & $\mathrm{G} 4$ & A5 & B5 & . $\mathrm{C}$ & $\mathrm{C} 4 \mathrm{I}$ & D4 & $\cdot$ & E4 & & \\
\hline Pitch-Level Accenting & & & $>$ & & & & $>$ & & & & $>$ & & & & & \\
\hline Pitch Metric & & & 14 & & & & 14 & & & $x$ & $(4$ & & & ) & & \\
\hline Amphib. Frame \& Anap. C & Cente & & $(1$ & 2 & )($. & (1 & 1 & 1 & 2 & )($. & $(1$ & 2 & & $(1$ & & \\
\hline Temporal Accenting & & & & $>$ & & $>$ & & & $>$ & & & $>$ & & & & \\
\hline Temporal Best Clock (We & ak) & & 13 & & & $(3$ & & & )$(3$ & & & $(3$ & & ) & & \\
\hline IOI Pattern of Accents & & & 1 & 2 & & 1 & 2 & - & 2 & . & 1 & 3 & . & - & & \\
\hline C. Temporary Dissonance & (Po: & lyrh & hyt & $\mathrm{hm})$ & & & & & & & & & & & & \\
\hline Pitch Pattern & $\mathrm{C4}$ & & E4 & $\cdot$ & & & E4 & $\cdot$ & F4 & G4 C & $c 4$ & $\cdot$ & D4 & $\mathbf{E 4}$ & $\mathbf{F} 4$ & . \\
\hline Pitch-Level Accenting & $>$ & & & & & $>$ & & & & & $>$ & & & & & \\
\hline Pitch Metric & 15 & & & & & $(5$ & & & & $x$ & 16 & & & & & ) \\
\hline Anapestic Rhythm & 1 & 1 & 2 & - & & 1 & 2 & • & 1 & 1 & 2 & - & 1 & 1 & 2 & • \\
\hline Temporal Best Clock & $>$ & & $>$ & & $>$ & & $>$ & & $>$ & & $>$ & & $>$ & & $>$ & \\
\hline LOI Pattern & 2 & . & 2 & • & 1 & 1 & 2 & & 2 & & 2 & & 2 & & 2 & \\
\hline
\end{tabular}

Figure 3. Patterns that are predicted to be disruptive to pitch-difference discrimination. Pattern $A$ is a case of out-of-phase consonance: pitch-level accenting is always out of phase with the temporal best clock. Patterns $B$ and $C$ are cases of rhythmic dissonance: pitch-level accenting and the temporal best clock divide the same total period with different length metrics.

and the same pitch pattern as in Figure 2. The main requirement for rhythmic dissonance is that there be accent clocks in different metrical bases that mutually divide the same temporal period. In most music this happens when a pattern in one metric is played by one voice and another pattern in another metric is played by another; the result is termed a polyrhythm. The listener can usually pay attention to one voice or the other but not both-except at very slow tempos (Handel \& Oshinsky, 1981).

We create a polyrhythm-type pattern in this example by having the temporal accenting that occurs regularly (nearly) every 3 intervals superimposed by a train of pitchlevel accenting every 4 intervals. Temporal accenting divides the period of 12 intervals into four equal parts; pitchlevel accenting divides it into three equal parts. Yeston
(1976) noted that the IOI "signature" of a polyrhythm is a "temporal retrograde"-one half of the IOI pattern is the mirror image of the other. Our example in Figure 2 would have a perfect retrograde if temporal accenting were perfectly regular every 3 pulses (e.g., 312213 instead of 1212213). Unlike the out-of-phase consonance example above, polyrhythms have a resting place and catch up with each other once every period they mutually divide. In our example the temporal best clock is in phase with the pitch-level accenting clock on the first note.

If a listener is to impose a perceptual clock that fits with grouping on the basis of pitch-level accenting and the contiguity of pitches in hertz, then he/she must ignore temporal accenting and grouping on the basis of the temporal contiguity of pitch onsets; the reverse is also true. The 
perceptual groups of temporal intervals that would be formed if a person could succeed in hearing this type of pattern in the metric of 4 or 3 are:

$\begin{array}{ccc}\begin{array}{c}\text { Dissonant } \\ \text { patterns }\end{array} & \begin{array}{c}\text { Grouping } \\ \text { (in metric base 4) }\end{array} & \begin{array}{c}\text { Grouping } \\ \text { (in metric base 3) } \\ 1\end{array} \text { 121 } 211121_{121211121} \\ 2 & 121112121 & 121112121\end{array}$

Both of the above patterns could support perceptual clocks in base 3 or 4 because they are ambiguous (see Povel \& Essens, 1985; Yeston, 1976); however, we assert that listeners will be more likely to impose a temporal metric of base 3 because of the way the patterns start and also because temporal accenting favors a best clock in 3 . If these patterns were recycled, the regular pitch-level accenting metric in 4 might help the listener hear the temporal metric in 4-but that is another experiment. We hypothesized that these rhythmically dissonant patterns would make pitch differences between two patterns as difficult to notice as in the case of out-of-phase consonance.

The last pattern in Figure 3 is similar to those tested by Deutsch (1980) and Boltz and Jones (1986). The temporal best clock is in base 2 . The pitch-level accent clock occurs irregularly, twice at a 5-interval and once at a 6interval distance. If we ignore the last 6 intervals where temporal and pitch-level clocks are in phase, the first 10 intervals are marked in the 101 pattern of accents as a $5 \times 2$ polyrhythm (221122). The pitch-level accent clock divides the period in half and the temporal best clock divides it into fifths. Boltz and Jones (1986) found that the number of pitch inflections or changes of pitch direction predicted the difficulty of transcribing such patterns. When accenting in the temporal pattern was metrically consonant with pitch-level accenting, that is, when the temporal pattern made inflections and unusual pitch skips predictable, pitch transcription was easier.

This result is clearly in line with our metrical consonance hypothesis and with the findings of Monahan and Carterette (1985), who had musicians rate pairs of melodies that varied in pitch pattern, rhythm pattern, or both. Listeners found temporal dimensions more important than pitch dimensions in determining pattern similarity. The pitch dimensions that were important were the overall melodic direction (up or down) and the number of pitch inflections; the hierarchical description of pitch level (i.e., pitch pattern rules) did not predict pattern similarity at all.

We note also, from a phenomenal view, how very different the same pitch pattern sounds played with different rhythm patterns (see Monahan \& Carterette, 1985, p. 1ff). Even for the simple pitch patterns in Figure 2, different rhythms group the onsets of different pitches together in time so it is sometimes difficult to hear even a +1 diatonic-scale-step pitch rule with an event periodicity of 3 notes. In the all-dactylic pattern the onsets of
D4, E4, and G4 are grouped together after the initial long $\mathrm{C} 4$ as:

\section{C4...D4 E4 G4 ... A5 B5 C4 ...D4 E4.}

The second example in Figure 2 begins with an anapest rhythm that clearly groups C4, D4, and E4. Particularly in the all-amphibrach pattern, there are large pitch skips between notes that are temporally close together-and this may lead to some degree of temporal incoherence and the identity of pitches may be harder to comprehend (van Noorden, 1975):

$$
\text { C4 D4...E4 G4 A5 ...B5 C4 D4... E4. }
$$

Furthermore, the accent on tones beginning the long temporal interval in each bar tends to stream together certain pitches across rhythmic groups; so the all-dactylic pattern emphasizes $\mathrm{C} 4, \mathrm{G} 4$, and $\mathrm{C} 4$; the all-amphibrach pattern emphasizes D4, A5, and D4. Just as pitches stream by their frequency, temporal intervals stream by the duration of tones in those intervals - and by this temporal streaming, the different patterns may emphasize very different modes and tonalities.

\section{Questions Raised by an Earlier Experiment}

Our research was specifically directed at questions raised by Jones et al. (1982) as to how a rhythmic context can "prime" recognition of pitch differences between patterns. First, we wished to know the source of priming. Jones et al. hardly questioned this: they combined several potential sources of temporal accenting in two contexts. The sources of accenting implied by their work are (1) length of temporal interval where longer IOIs provide accent, (2) a pause or rest before the to-be-recognized pitch, and (3) the TDI within the IOI. Second, we wished to know to what extent rhythm in one part of a pattern could act as an enhancer or distractor for pitch recognition in another part of the pattern. Are rhythmic effects on pitch-difference discrimination only local effects that lengthen the temporal interval or duration of the to-berecognized pitch or cause a rest or pause to occur before the to-be-recognized pitch? Jones et al. left this question unanswered also: the context preceding one changed-pitch location was metrically regular and that preceding the other was not.

We will outline the findings of Jones et al. (1982) in some detail in order to make apparent the reasons for the design of our own experiment. Jones et al. played each of several 9-sequential-tone melodies that differed in the complexity of their pitch structures together with each of four different rhythms: isochronous, dactylic, anapestic, and irregular. Listeners heard standard-comparison pairs of such patterns in which the pitch was changed for tones at serial position (SP) 4 or at SP6. According to Jones et al., tones at SP4 were less "codable" than those at SP6 because they instantiated a "higher-order pitch rule," by which they meant that an unusual pitch skip preceded the 
tone initiating the phrase starting at SP4 or a contour inflection followed it; in other words, tones at SP4 were points of pitch-level accent. Let us examine these rhythmic contexts: the short tones in all rhythms had a duration of $250 \mathrm{msec}$ followed by a silent interval of $50 \mathrm{msec}$; the long tones had a duration of $500 \mathrm{msec}$ followed by a silent interval of $100 \mathrm{msec}$; the rests, denoted $R$, were 300 -msec silent intervals. Where $\mathrm{A}=$ accented and $\mathrm{U}$ = unaccented,

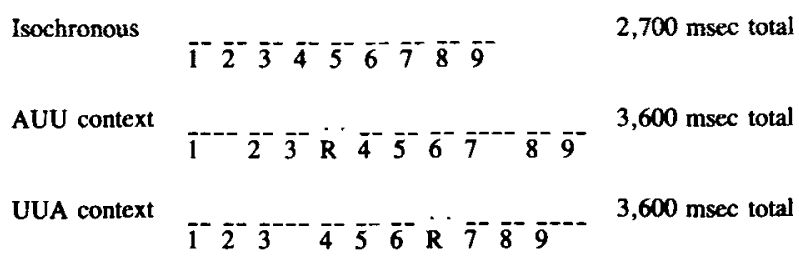

Jones et al. (1982) stated about the AUU and UUA sequences:

Two important points to note about these rhythms are: (a) both embed the same central tone triplets, with the same thing, as found in the isochronous pattern; and (6) the two patterned rhythms differ with respect to temporal locations of accents (the UUA context induces an accent on the sixth tone of the common central segment, whereas the AUU induces an accent on the fourth tone of this segment). (p. 214, italics ours)

Listeners recognized pitch changes significantly better at SP4 in the dactylic (AUU) and isochronous contexts than in the anapestic (UUA) or irregular contexts. On the other hand, at SP6, the anapestic context provided only slightly better recognition.

We were perplexed because the so-called AUU or dactylic context does not sound like a dactylic rhythm; instead, there are major natural temporal accents on the first, third, and seventh tones, and perhaps (depending on the listener) a lesser accent on the fourth tone-AUAaUUAUU, but certainly not AUUAUUAUU. The silent rest interval of the AUU context "attaches" itself to the preceding sound-filled interval to make a long IOI between tones 3 and 4 of the sequence, and the third tone is definitely more temporally accented than the fourth. The IOI pattern for the AUU context is 212111211 , which is somewhat irregular metrically. The temporal accent that the fourth tone receives may be due both to the preceding silence and to the fact that it begins the first of a group of 3 or more intervals-a position of natural temporal accent, according to Povel and Okkerman (1981).

On the other hand, for the UUA context Jones et al. (1982) did seem to assume that the silent rest interval attaches itself to the preceding sound-filled interval, making the tone at SP6 sound temporally accented. This pattern definitely sound like an anapest rhythm with an IOI pattern of 112112112. The assumption here, which is one that is typically made in the literature, is that the TDIs within the IOIs of a temporal pattern make little or no difference to the perception of rhythm. Both Monahan (1984) and Gabrielsson (1985) raised the question of the importance of the degree of interval-filling; both noted that interval-filling is the basis for the legato/staccato distinction, a distinction that has not received much scientific study. We suggest that the degree of interval-filling can form attentional streams, so that if two instruments are playing the same sequence of frequencies, on in staccato fashion and the other legato, the listener can still attend to one or the other. Or, even within the same piece played by a single instrument, tones played in legato fashion may form one stream while tones played in staccato fashion form another. Our question with regard to temporal interval-filling is whether a pattern such as $112111 R 112$ (where $R$ denotes a one-pulse silence) is as effective at promoting pitch-difference detection at the sixth note of such a series as a pattern that has a relatively sound-filled interval at that position. The same question can be asked about dactyl or amphibrach patterns: Does a pattern that includes a "rest dactyl" (e.g., 211 IR11 211) promote recognition at the fourth tone of the series as well as does the pattern 211211211 , which has a sound-filled interval at that position? Unlike Jones et al.'s irregular AUU context pattern, our rest-dactyl center rhythm is temporally regular with regard to its onset-to-onset pulse interval pattern, which is 211 , and when played with a surrounding dactyl pattern, it sounds dactylic. We are interested in whether either of these patterns is better than Jones et al.'s AUU context in aiding pitch-difference detection for the fourth tone of the series. Our hypothesis is that the metrically regular soundfilled intervals will tend to aid detection of pitch change the most.

As to the question of whether effects of temporal accenting on pitch difference recognition are only local ones, we tested whether rhythmic patterning in one part of a sequence has main effects or interactions with rhythmic patterning in another part of the sequence on the ability to recognize pitch differences at various SPs in the pattern. In some of the above examples, if an anapestic rhythm is best (at SP6) only when preceded by an anapestic rhythm and a dactylic rhythm is best (at SP4) only when preceded by a dactylic rhythm, there should be a significant interaction of preceding with following rhythms (when each may occur followed either by itself or by the other) on the ability to detect pitch changes at particular SPs.

\section{OVERVIEW OF THE DESIGN}

The present study, designed to assess the discriminability of a single pitch chroma change between two musical patterns as a product of pitch and temporal patterning, employed a complete mixed factorial design. Musical experience (musician or nonmusician) was the only between-groups factor. Twenty-one different rhythmic 
patterns combined with each of four different melodies to form the 84 standard stimulus patterns. Every listener heard each standard paired with 10 comparison patterns, resulting in 840 trials per listener. Half of the comparison patterns were identical to the standard; the other half had a single pitch change that occurred at one of five different serial positions.

\section{Timing Pattern Construction}

In each sequence, the first and last groups of three pitches were played with the same timing pattern, which could take one of three forms: dactyl, anapest, or amphibrach. We called these rhythmic frames, because they bracketed the timing patterns of the center 3 notes. The 3 center pitches of the 9-tone pattern were played with seven different timing patterns called center rhythms. Figure 4 shows the music notation for the resulting 21 temporal patterns; each of the two parts of a frame and each center rhythm may be thought of as lasting 4 eighth notes, with note onsets at only three of the four equalinterval locations.

Table 1 shows the labels and time values for the three frames and seven center rhythms. We have used for the frames and center rhythms labels that traditionally have been applied to poetry; we have also employed labels that represent patterns in terms of successive IOIs. We assume, along with Povel and Essens (1985), that temporal accents are heard primarily on tones with longer IOIs (or as Povel \& Essens put it, "on relatively isolated tones") and that the other notes in the patterns are relatively unaccented.

In order to discover whether the priming effect of temporal accenting is a function only of the IOI (the time of the temporal event) or also of the TDI within the IOI, we created two versions of the dactyl, anapest, and amphibrach center rhythms, as shown in Table 1. In the "rest" version of these patterns, the tone duration in the long IOI is $250 \mathrm{msec}$, or $42 \%$ of the IOI; in the "filled" version, the tone duration is $83 \%$ of the $\mathrm{IOI}$, as it is for all other tones in the sequences. The contrast between these versions may also be considered the difference between the playing of a single note in the sequence in a relatively staccato (short duration) or legato (long duration) fashion while holding IOI constant. In our naming of these patterns, we have assumed that the time of the rest interval attaches itself to the time of the preceding sound-filled interval, creating a lengthened IOI and therefore a temporal accent.

We have also included among the center rhythms a pattern that we term $R+3 / 8$. When this center rhythm is combined with a dactylic frame, it is identical to what Jones

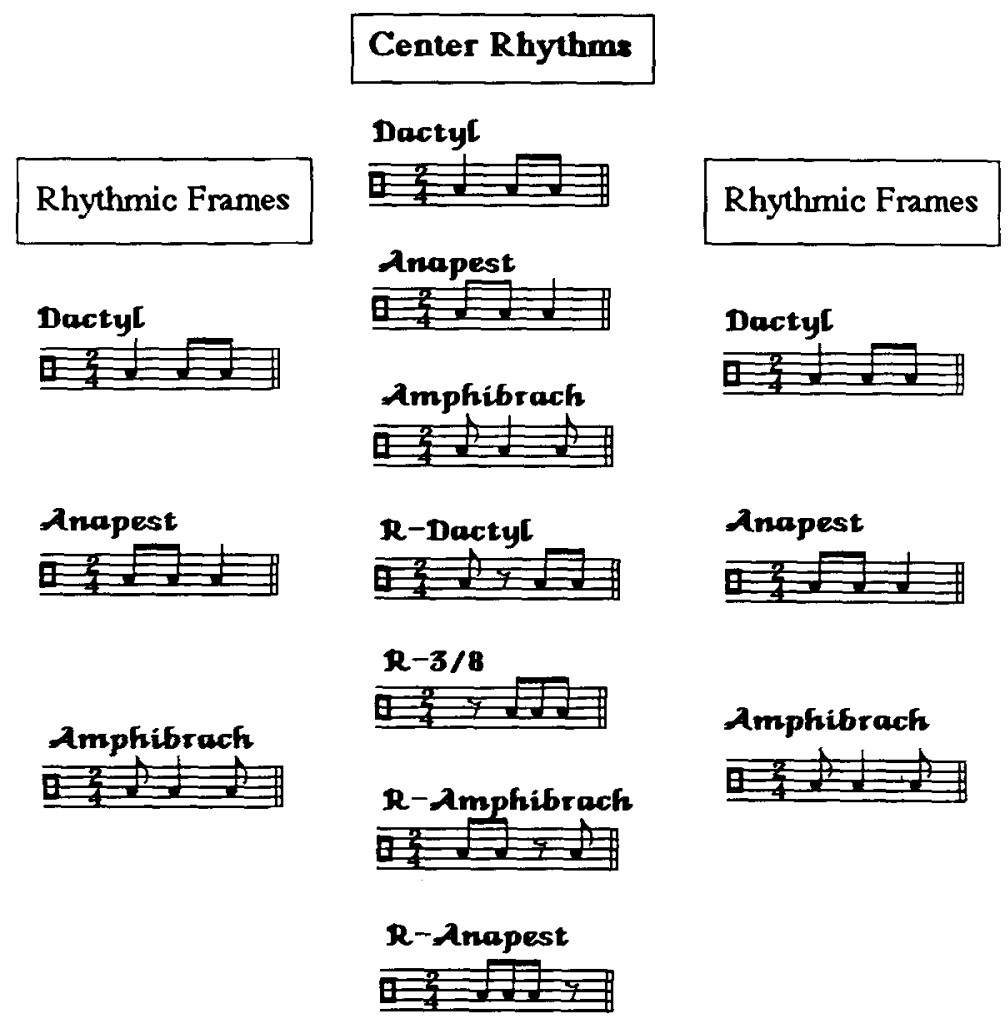

Figure 4. Musical notation for rhythmic frames and center rhythms. Rhythmic frames are the temporal patterning for tones at serial positions, 1, 2, and 3, and 7,8 , and 9 of experimental stimuli. Center rhythms provide the temporal patterning of tones 4, 5, and 6 of experimental stimuli. 
Table 1

Temporal Patterns Employed in the Experiment

\begin{tabular}{llll}
\multicolumn{1}{c}{ Name } & $\begin{array}{c}\text { Accent } \\
\text { Pattern }\end{array}$ & $\begin{array}{c}\text { Beat } \\
\text { Label }\end{array}$ & Time Pattern* (msec) \\
\hline \multicolumn{4}{c}{ Rhythmic } \\
Frames for Tone Serial Positions 1-3 and 7-9 \\
1. Dactyl & AUU & 211 & $500(100), 250(50), 250(50)$ \\
2. Anapest & UUA & 112 & $250(50), 250(50), 500(100)$ \\
3. Amphibrach & UAU & 121 & $250(50), 500(100), 250(50)$
\end{tabular}

Center Rhythms for Tone Serial Positions 4-6

\begin{tabular}{|c|c|c|c|}
\hline 1. Dactyl & AUU & 211 & $500(100), 25$ \\
\hline 2. Anapest & UUA & 112 & $50(50), 250(50), 500(100)$ \\
\hline 3. Amphibrach & UAU & 121 & $50(50), 500(100), 250(50)$ \\
\hline 4. Rest-Dactyl & AUU & $\operatorname{lR} 11_{\dagger}^{\dagger}$ & $250(50+300=350), 250(50), 250(50)$ \\
\hline 5. Rest-Anapest & UUA & $11 R+$ & $250(50), 250(50), 250(50+300=350)$ \\
\hline Amphibrach & UAU & $1 \uparrow$ & \\
\hline & & & \\
\hline \multicolumn{4}{|c|}{$\begin{array}{l}\text { Note-A }-\mathrm{A} \text { accented; } \mathrm{U}=\text { unaccented. } \text { *Each interonset interval (IOI) consists of } \\
\text { a sound-filled tone duration interval (values outside parentheses) plus a silent interval } \\
\text { (values inside parentheses). } † \text { The IOI notation for } 1 \mathrm{R} 11,111 \mathrm{R} \text {, and } 11 \mathrm{R} 1 \text { would } \\
\text { most properly be } 211,112 \text {, and } 121 \text {, respectively; hence the names rest-dactyl, rest- } \\
\text { anapest, and rest-amphibrach. } \$ \text { There is no IOI notation that applies to the } \mathrm{R}+3 / 8 \\
\text { center rhythm pattern alone, because the time of the initial rest will be added to the } \\
\text { time of the note in the third serial position (see text). }\end{array}$} \\
\hline
\end{tabular}

et al. (1982) called their $A U U$ or dactylic context. The IOI pattern of this center rhythm combined with each of the three rhythmic frames is as follows:

Dactylic frame plus $R+3 / 8$

Anapestic frame plus $\mathrm{R}+3 / 8$

Amphibrach frame plus $\mathrm{R}+3 / 8$

2121111211

111311111112

1221111121

\section{Standard Pitch Patterns}

We employed four 9-note pitch patterns that differed with regard to two variables: melodic rule ( 2 levels) $\times$ type of pitch-level accenting ( 2 levels). The melodic notation for these patterns is shown in Figure 5; Figure 6 shows the melodic contour of the melodies where a plus represents ascending motion and a minus represents descending motion between consecutive notes.

The four melodies are the same as those used by Jones et al. (1982, p. 212) for "one-rule" and "two-rule" melodies. The melodies may be described as three 3-tone segments. The successive pitch intervals within each segment follow either $a+1$ or -1 diatonic-scale-step rule. In one-rule melodies (Melodies 1 and 2) the third segment is a repetition of the first segment, which is not the case for two-rule melodies (Melodies 3 and 4). Earlyinflected melodies (Melodies 2 and 4) have a pitch inflection between SP4 and SP5, which creates a pitch-level accent for the tone at SP4; Melodies 1 and 3 have an unusual pitch skip ( +3 semitones) between SP3 and SP4, which also creates a pitch-level accent at SP4. These patterns will be referred to as early pitch-skip melodies. At SP4 we have a differential test of the detectability of a pitch change as a product of two different kinds of pitchlevel accenting: at the point of a unidirectional unusual pitch skip (Melodies 1 and 3) or at the point of a highly predictable pitch contour inflection without a preceding pitch skip (Melodies 2 and 4). Beyond this, we note that all melodies have strong pitch-level accenting at SP7 (following Thomassen's [1982] results); this is due to large preceding pitch skips in Melodies 1, 3, and 4 and to following pitch inflections in Melodies 1 and 2.

\section{Comparison Pattern Construction}

For each of the 84 standard patterns formed by the crossing of 4 melodies with 21 rhythms, we generated five comparison patterns that changed the pitch of a single chroma of the standard. All comparisons were raised an octave above the standard. The set of all deviant comparison melodies is presented in Figure 7; the notation in this figure is for pitches 1 octave below what listeners heard. The changed pitch occurred at SPs 3, 4, 5, 6, and 7 on five different trials. Additionally, for each standard there were five trials in which there was no pitch change.
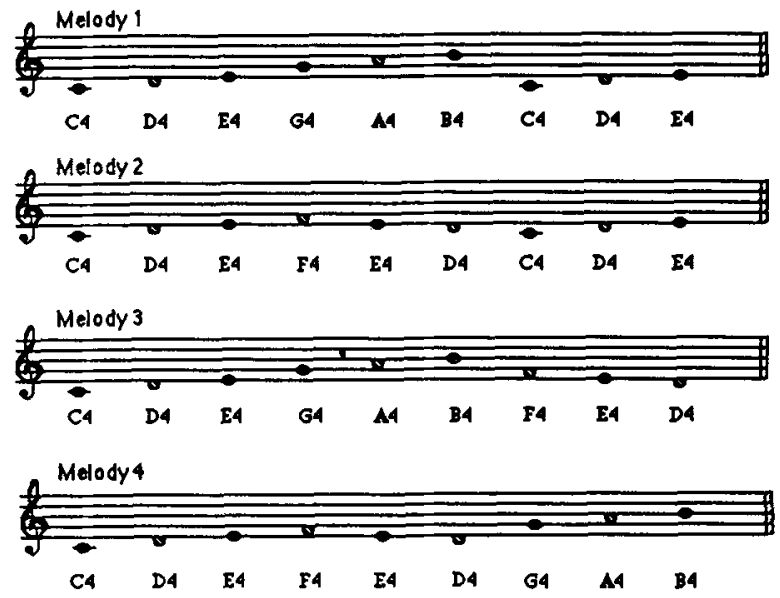

Figure 5. Musical notation for the four pitch patterns of the experimental stimuli. 
Pitch Contour

\begin{tabular}{|c|c|c|c|c|}
\hline Melody 1 & +4 & $+t$ & ++ & 5 \\
\hline Melody 2 & + & -- & ++ & $3,5,6$ \\
\hline Melodg 3 & $+t$ & $+t$ & -- & 5 \\
\hline Melody 4 & ++ & --1 & ++ & 3,5 \\
\hline
\end{tabular}

a Serial position(s) which alter contour when pitch is changed from the standard.

Figure 6. Pitch contours of the four pitch patterns of the experimental stimuli. A plus sign means an increase in pitch level between successive tones and a minus sign means a decrease in pitch level between successive tones.

By comparing listeners' ratings when there was a pitch change and when there was no change we could measure the discriminability of the patterns as a product of pitch and timing variables.

In contrast to the present experiment, Jones et al. (1982) examined the discriminability of pitch changes at only SPS
4 and 6 . In constructing the deviant tone melodies, they employed the following constraints:

(1) Deviant tone sequences were not permitted to break the contour established by the standard sequence. (2) No deviant tone was repeated in succession in the nine-tone sequence. (3) Deviant tones from the $\mathrm{C}$ major triad ( $\mathrm{C}, \mathrm{E}$, G) were avoided due to their high musical familiarity. (4) All deviant tones were the $\mathrm{C}$ major scale. (5) Changed tones could not create a repeating tone. (6) Deviations were between 1 and 3 scale units. (p. 214)

We found that we could not apply these constraints to Melody 2 (which is also one of Jones et al.'s pitch patterns) at SP6 without causing a change in melodic contour from the standard. The descending motion between SP5 and SP6 can be preserved only by altering the pitch $D$ at SP6 to the B below; this, in turn, alters the descending motion between SP6 and SP7 to ascending motion (cf. Figures 5 and 7 for Melody 2 at SP6). In addition to the inevitable contour break for Melody 2 when a pitch is changed at SP6, in the present experiment a pitch contour break occurs for all melodies at SP5, because alteration of a tone at this point occurs in the middle of a lowerorder pitch rule. Also, early-inflected Melodies 2 and 4
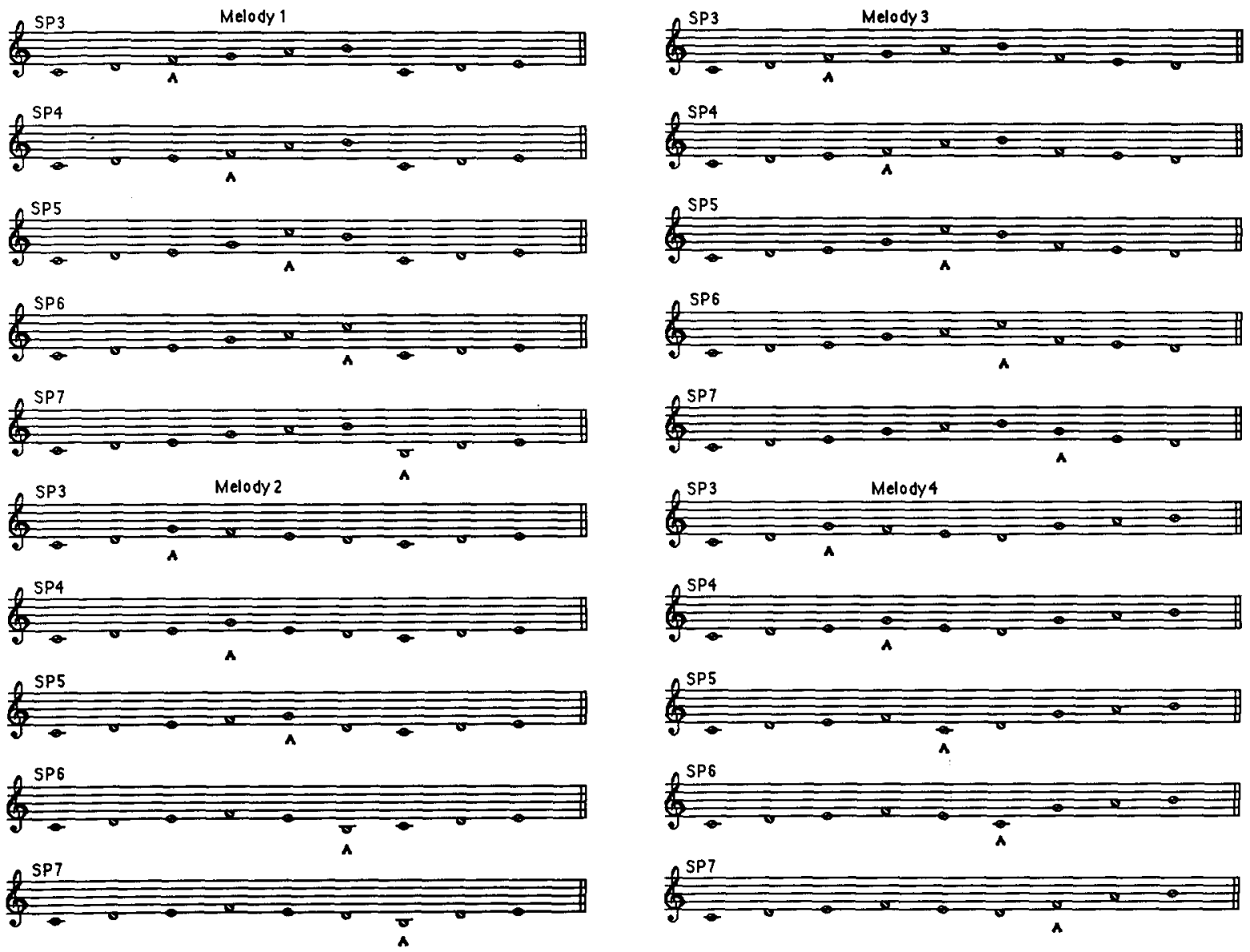

Figure 7. Musical notation for all deviant chroma comparison melodies. Altered pitch is marked at each serial position at which change occurred (serial positions $3,4,5,6$, and 7 ) for each of four experimental standard melodies. All comparison melodies were played 1 octave higher than shown in notation. 
had different contours from the standard when the tone at SP3 was altered. We note these contour breaks because good performance should result whenever standard and comparison patterns differ in pitch contour (Dowling \& Fujitani, 1971).

We therefore followed all of the constraints above wherever possible; however, we decided to permit deviant tones of the $\mathrm{C}$ major triad in order to provide minimal disjunct motion in the resulting contour. The construction of deviant melodies according to these guidelines was completely deterministic; that is, no arbitrary selection of deviant tones was possible.

\section{Predictions for Various Serial Positions \\ Based on Rhythmic Variables}

We hypothesized that relatively longer sound-filled intervals would provide for better pitch-difference discrimination for tones at various serial positions. We refer to this as the long filled-interval attentional beacon hypothesis. This means that patterns with anapest frames should give the best discrimination performance at SP3 and patterns with dactyl frames should give the best performance at SP7. Patterns with filled-dactyl center rhythms (211) should be best at SP4; patterns with filled-amphibrach center rhythms (121) should be best at SP5; and patterns with filled-anapest center rhythms at SP6 (112). We note that tones at SPs 3 and 7 have less temporal variabiity and a greater likelihood of initiating a long filled interval than those at SPs 4, 5, or 6, which may result in better performance on SPs 3 and 7.

If there are main effects of frames on SPs governed by center rhythms (or vice versa), they should favor dactylic and anapestic rhythms, because these are patterns that are rhythmically consonant with pitch-level accenting. If there are interactions of frames with center rhythms and SPs, then dactylic frames should be best with dactylic center rhythms (especially at SP4) and anapestic frames should be best with anapestic center rhythms (especially at SP6).

We hypothesized that amphibrach frames and center rhythms would give subjects the least certainty of the grouping of pitches by a simple +1 or -1 diatonic-scalestep rule and would result in poor performance. We refer to this as the nonconsonance hypothesis. In contrast to Jones et al.'s (1982) predictions (see below), we hypothesized that there would be no difference in pitch difference detectability at SPs 4 and 6 overall. Long filledinterval accenting occurs equally often at both SPs. Performance at these SPs should be equally poor with amphibrach center rhythms; nonmusician listeners are not likely to recognize the simple +1 or -1 pitch rule when they hear it because nonconsonant temporal grouping will tend to override the pitch grouping.

\section{Predictions Based on Redundancy and Pitch Rules}

One-rule melodies have relatively high redundancy, because the last 3 tones are identical to the first 3 , which is not the case for the two-rule melodies. We hypothe- sized that the last 3 pitches might provide a reference for other pitches in the same melody; therefore, one-rule melodies might be easier than two-rule melodies. Jones et al. (1982) made the same prediction, for slightly different reasons; they assumed that the successive application of the same rule within their system of rules, as opposed to the application of two different rules within that system, had some psychological reality for the listener.

Jones et al. (1982) made a second prediction with regard to redundancy:

Lower order rules that relate tones within a three-tone segment... have greater regularity (i.e., frequency) than do higher order rules that relate tones between three-tone segments. ... Note, then, that a tone occurring at the sixth serial position (SP6) completes a lower order rule, whereas one at the fourth position (SP4) instantiates a higher order rule. (p. 213)

Jones et al. (1982) argued further that the difference in pitch-rule redundancy between lower- and higher-order rules should make pitch changes at SP4 much harder to recognize than pitch changes at SP6.

We made no strong prediction concerning subjects' performance with early-inflected and early-pitch-skip melodies. Such a prediction would involve only SP4 and the salience of pitch change for the two different kinds of pitch-level accenting. On the one hand, Thomassen (1982) showed that pitch inflection creates a more salient accent than does a unidirectional pitch skip, which suggests that performance would be best for Melodies 2 and 4 . On the other hand, Boltz and Jones (1986) demonstrated that the number of inflections is negatively correlated with the listener's ability to transcribe a melody accurately, which suggests that performance might be best for Melodies 1 and 3 . The seemingly conflicting prior evidence left us no basis for a hypothesis.

\section{METHOD}

\section{Subjects}

There were 22 subjects; of these, 20 participated in the experiment in partial fulfillment of the requirements for an introductory psychology course at UCLA. There were 12 musicians (defined as having 5 or more years of formal music training) and 10 nonmusicians. For purposes of analysis, the two groups were equated in size by randomly discarding the responses of 2 musicians.

\section{Stimulus Generation and Trial Construction}

A Synclavier II digital computer-controlled synthesizer was used to generate the stimulus series. All tones were 16-component square waves, which were utilized to minimize the frequency/loudness interactions that are characteristic of sine tones. An exponential attack envelope of $40 \mathrm{msec}$ was applied to each tone.

Each of 84 standard patterns ( 21 thythms $\times 4$ melodies) was paired with five octave-raised but otherwise identical comparison patterns and also with five comparisons in which there was a pitch change at a single SP from SP3 to SP7. This resulted in 840 trial items.

Trials comprised (1) a $4000-\mathrm{Hz}, 750-\mathrm{msec}$ warning tone; (2) 250 msec silence; (3) the standard pattern $(3.6 \mathrm{sec})$; (4) the interstimulus interval $(900 \mathrm{msec})$; (5) the comparison pattern 
( $3.6 \mathrm{sec}$ ); and (6) a $3-\mathrm{sec}$ response period. Every $21 \mathrm{st}$ item was followed by a 30 -sec rest period. The 840 trial items were randomly ordered and output as eight sets of 105 items; each set was monophonically recorded on a separate Maxell UD Metaxial tape on a Fostex X-15 cassette using Dolby noise reduction and metal tape equalization.

\section{Procedure}

Subjects were run singly in a soundproof room. A Fostex X-15 cassette recorder reproduced the stimulus tapes through headphones. The steady-state signal at each earphone was measured at $70 \mathrm{~dB}$ SPL $(+.5 \mathrm{~dB})$. Each subject received a randomly selected ordering of the eight stimulus tapes. An IBM PC computer collected subject responses and unscrambled the random ordering of items for data analysis. Seven consecutive keys on the computer keyboard were labeled from 1 (most similar) to 7 (least similar). The computer graphically displayed these keys and highlighted the keypress selected by the subject. The computer also prompted subjects' responses according to the timing of the stimulus series.

The following instructions were read to each subject:

This is an experiment to test your perception of melodic similarity. On each trial you will hear two melodic patterns which are preceded by a very high warning tone. If the two patterns seem very similar to you, press 1 on the keyboard; if they seem very different, press 7. The range in between, from 2 to 6 , signifies decreasing similarity as you go from smaller to larger numbers. For example, a response of 1 tells us that you think the patterns were very similar; a response of 3 tells us that they seemed somewhat similar; a response of 4 tells us that there was an intermediate degree of similarity; a response of 5 tells us that you think the patterns were somewhat different; a response of 7 indicates that you think the patterns were not very similar. You must respond on every trial, otherwise the recorded patterns and computer record of your responses will be out of synchrony and your data will be unusable. Altogether, you will hear 840 pairs of patterns. Try to use all seven categories from most similar to least similar during the experiment.

Before the beginning of the first session, each subject received 21 practice trials selected at random from the set of 840 trials. Experimenters monitored performance on these trials to ascertain that subjects comprehended the task. Each subject was run in four separate sessions, scheduled at the subject's convenience. All subjects completed the experiment within a 2-week period.

\section{RESULTS}

The results of this study describe the performance of listeners in discriminating patterns that might be either the same or different with respect to the pitch chroma of one of the elements. The analysis was done in two stages. In the first, individual subjects were replicates for each of the main effects and interactions. However, for each combination of pitch and rhythmic variables, individual subjects heard only a single pattern in which pitch was changed at a particular SP; that is, SP of pitch change was not a repeated measure within subjects for each of the combinations of pitch and rhythmic variables. Therefore, in the individual-subjects analysis, the measure of sensitivity to change was collapsed over the five SPs at which change occurred. This, of course, did not allow us to look at sensitivity to change at particular SPs, or at the interaction of variables at particular SPs. We present the individual-subjects analysis primarily as corroboration of the validity of the second, "supersubjects" (Crowder, 1982; Samuel, 1981), analysis.

\section{Individual-Subjects Analysis}

We calculated areas under the memory operating characteristic (MOC; Clarke, 1964) separately for each subject for each of 84 conditions ( 3 rhythmic frames $\times$ 7 center rhythms $\times 2$ melodic rules $\times 2$ types of pitchlevel accenting), using the six criteria possible with a 7point rating scale (McNicol, 1972). Area under the MOC is used as an unbiased estimate of the proportion of correct responses, where chance would be .50 . For each condition we derived the "hit" distribution from the listener's responses to five trials in which there were pitch differences. For each condition we derived the "false alarm" distribution from the listener's responses to five trials in which there were no pitch changes. Since there was only one trial per condition per SP of pitch change for each subject, hits were collapsed over SPs. Therefore, the present analysis is for sensitivity to pitch differences summed over the five possible SPs in which the pitch change occurred.

The MOC values for each listener in each of the 84 conditions were subjected to a split-plot analysis of variance (ANOVA) with musical experience as the between-groups variable and rhythmic frame, center rhythm, melodic rule, and type of pitch-level accenting as repeated measures. Under the assumption that population variances and covariances of treatment levels are equal, univariate tail probabilities are appropriate. In repeated measures analysis, this assumption is often not met, and the Geisser-Greenhouse conservative $F$ test (Geisser \& Greenhouse, 1958), which adjusts degrees of freedom depending on the heterogeneity of variances and covariances, may be deemed more appropriate. Both are reported, when available, for all main effects and for interactions that are significant at $p<.05$ by both univariate and Geisser-Greenhouse tests. By these criteria, the ANOVA showed that four of the five independent variables had significant main effects and that there was a significant interaction (see Table 2). All post hoc tests were Tukey $a$ procedures with a constant experimentwise alpha level of .05. Main effects were analyzed using the procedure outlined by Linton and Gallo (1975); interactions were analyzed for unconfounded means from an interaction table (Cicchetti, 1972; Linton \& Gallo, 1975). Each of these results will be discussed in turn.

Table 2

Main Effects and Significant Interactions of Musical Variables on Individual Listeners' Sensitivity to Pitch Change

\begin{tabular}{lrrcc}
\hline Variable & \multicolumn{1}{c}{$F$} & $d f$ & $\begin{array}{c}\text { Univariate } \\
\text { Probability }\end{array}$ & $\begin{array}{c}\text { Geisser-Greenhouse } \\
\text { Probability }\end{array}$ \\
\hline EX* & 6.10 & 1,18 & .0238 & \\
FR & 11.03 & 2,36 & .0002 & .0006 \\
CR & 2.03 & 6,108 & .0678 & .0948 \\
MR & 22.73 & 1,18 & .0002 & \\
PLA & 16.83 & 1,18 & .0007 & \\
FR $\times$ & & & & \\
PLA & 3.44 & 2,36 & .0431 & .0441 \\
\hline
\end{tabular}

Note-Dependent variable is area under the memory operating characteristic, Abbreviations: $\mathrm{EX}=$ experience, $\mathrm{FR}=$ rhythmic frame, $\mathrm{CR}=$ center rhythm, MR = melodic rule, PLA = type of pitch-level accenting. *Experience is a between-groups variable; other variables are repeated measures. 
Musical Experience. Musicians detected pitch differences significantly better than did nonmusicians (average MOC values of .944 and .856 , respectively). There was no evidence of significant interaction of musical experience with any other variable.

The musicians averaged 7.3 years of formal training $(S D=2.3$ years); nonmusicians averaged 1.3 years of formal training ( $S D=2.0$ years). Individual-subject MOCs averaged over the 84 conditions of the present analysis were correlated with years of formal musical training. The correlation for musicians was $.68, d f=8$, $p<.05$; the correlation for nonmusicians was .12 , $d f=8$, which was not significant.

Rhythmic variables. There was a highly significant effect of rhythmic frame. Post hoc analysis showed that sensitivity to pitch differences was similar for patterns with anapestic and dactylic frames, and that performance with both of these pattern types was significantly better than that with amphibrach frames (average MOC values were $.913, .905$, and .883 , respectively; see Table 3 ).

Although the main effect for center rhythms only approached significance (see Table 2), it showed similarity to the result for rhythmic frames: within each level of musical experience, pitch recognition performance with dactylic and anapestic center rhythms was better (with one exception-musicians' response to amphibrach 121 center rhythm) than performance with amphibrach rhythms (see Table 4).

A somewhat unexpected result was performance with the $\mathrm{R} 111$ center rhythm; this pattern is slightly metrically irregular when combined with rhythmic frames, yet pitch discrimination performance was moderately good.

Table 3

Listeners' Mean Sensitivity to Pitch Change for Levels of Musical Experience and Different Rhythmic Frames

\begin{tabular}{lccccc}
\hline & \multicolumn{4}{c}{ Type of Rhythmic Frame } \\
\cline { 2 - 5 } Experience & Dactylic & Anapestic & Amphibrach & Mean \\
\hline Musicians & .948 & .959 & .926 & .944 \\
Nonmusicians & .863 & .866 & .840 & .856 \\
Mean & .905 & .913 & .883 &
\end{tabular}

Note-Dependent variable is area under the memory operating characteristic; chance performance is .50 .

Table 4

Listeners' Mean Sensitivity to Pitch Change for Different Center-Phrase Rhythmic Patterns

\begin{tabular}{|c|c|c|c|c|c|c|c|c|}
\hline \multirow[b]{3}{*}{ Experience } & \multicolumn{8}{|c|}{ Center-Phrase Rhythmic Patterns* } \\
\hline & \multicolumn{2}{|c|}{ Dactylic } & \multicolumn{2}{|c|}{ Anapestic } & \multicolumn{2}{|c|}{ Amphibrach } & \multirow{2}{*}{$\frac{\mathrm{R}+3 / 8}{\mathrm{R} 111}$} & \multirow[b]{2}{*}{ Mear } \\
\hline & 211 & $1 R 11$ & 112 & $111 R$ & 121 & $11 \mathrm{R} 1$ & & \\
\hline Musicians & .942 & .938 & .946 & .955 & .941 & .935 & .952 & .944 \\
\hline Nonmusicians & .877 & .867 & .857 & .846 & .854 & .829 & .864 & .856 \\
\hline Mean & .910 & .903 & .901 & .900 & .898 & .882 & .908 & \\
\hline
\end{tabular}

Note-Dependent variable is area under the memory operating characteristic; chance performance is .50. *Rhythmic pattern notation is interpreted as follows: 2 is a sound-filled interval with the duration of a quarter note; 1 is a sound-filled interval with the duration of an eighth note; $R$ is a rest or silent interval with the duration of an eighth note.
Table 5

Listeners' Mean Sensitivity to Pitch Change for Levels of Musical Experience and Different Melodic Rules

\begin{tabular}{lccc}
\hline & \multicolumn{3}{c}{ Melodic Rule } \\
\cline { 2 - 4 } Experience & 1-Rule & 2-Rule & Mean \\
\hline Musicians & .956 & .932 & .944 \\
Nonmusicians & .872 & .841 & .856 \\
Mean & .914 & .886 & \\
\hline
\end{tabular}

Note-Dependent variable is area under the memory operating characteristic; chance performance is .50 .

Table 6

Listeners' Sensitivity to Pitch Change for Levels of Musical Experience and Types of Pitch-Level Accenting

\begin{tabular}{lccc}
\hline & \multicolumn{3}{c}{ Type of Pitch-Level Accenting } \\
\cline { 2 - 4 } Experience & $\begin{array}{c}\text { Early } \\
\text { Inflection }\end{array}$ & $\begin{array}{c}\text { Early } \\
\text { Pitch Skip }\end{array}$ & Mean \\
\hline Musicians & .968 & .920 & .944 \\
Nonmusicians & .880 & .832 & .856 \\
Mean & .924 & .876 & \\
\hline
\end{tabular}

Note-Dependent variable is area under the memory operating characteristic; chance performance is .50 .

Averaged over all contexts, patterns that mark temporal accent with a continuous sound-filled interval $(211,112$, and 121) tended to evoke about the same pitch discrimination as their counterparts in which accents are marked by an eighth note followed by a rest $(1 \mathrm{R} 11,111 \mathrm{R}$, and 11R1).

Pitch variables. The main effect for melodic rule was highly significant: one-rule melodies in which the third melodic phrase repeats the first phrase resulted in better discrimination of pitch (mean MOC value $=.914$ ) than did two-rule melodies $($ mean $\mathrm{MOC}$ value $=.886$; see Table 5). This result corroborates the same finding by Jones et al. (1982).

Sensitivity to pitch change was significantly better for patterns in which pitch-level accenting took the form of an early inflection rather than an early unidirectional pitch skip. Early-inflected and pitch-skip patterns had average MOC values of .924 and .876 , respectively (see Table 6).

Interaction of rhythmic and pitch variables. There was a significant interaction of rhythmic frame and type of pitch-level accenting on sensitivity to pitch change, which is shown in Figure 8 for both musicians and nonmusicans. With early-inflected melodies, both groups showed only slightly better pitch-discrimination performance for anapestic and dactylic frames than for amphibrach frames. With early-pitch-skip melodies, there was a definite ordering of performance by type of rhythmic frame: averaged over both groups, the MOC value for anapestic, dactylic, and amphibrach frames were .898, .878 , and .852 , respectively. Post hoc analysis revealed that only the difference between performance with anapestic and that with amphibrach frames was significant. Apparently, anapestic frames helped listeners organize the two early-pitch-skip patterns so that pitch changes were better recognized. However, with the present analysis we 


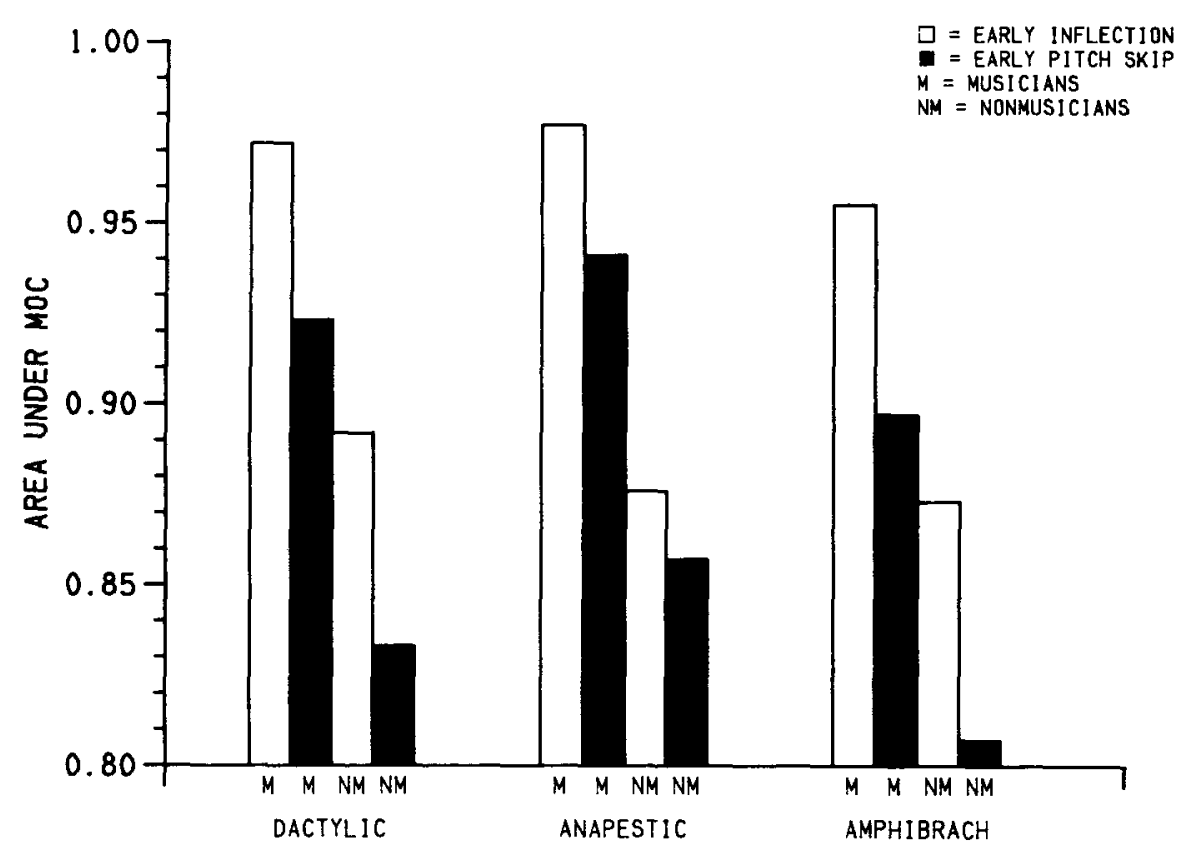

RHYTHMIC FRAME

Figure 8. Individual subjects' sensitivity to pitch differences for different degrees of musical experience, different rhythmic frames, and different types of pitch-level accenting early in. The measure of sensitivity is area under the memory operating characteristic (MOC), where chance performance is $\mathbf{. 5 0}$.

were not able to assess whether this effect or any of the previously discussed main effects was a function of serial position of pitch change in the patterns.

\section{Supersubjects Analysis}

For the second analysis, we created 4 supersubjects (Crowder, 1982; Samuel, 1981), 2 each within each of the musical experience groups. We did this by matching five pairs of subjects within each group on the basis of musical experience and assigning 1 member of each pair to 1 of 2 supersubjects. Each supersubject's sensitivity to pitch differences was based on the combined performance of 5 different individuals. For supersubjects, SP of pitch change was a repeated measure within subjects for each of the combinations of pitch and rhythmic variables. We can, therefore, look at the effects of pitch and rhythm and their interactions at each SP.

For each of the 4 supersubjects, the MOC values for each of 420 conditions ( 84 conditions of the previous analysis $\times 5$ serial positions of pitch change) were calculated in the following manner. Each hit distribution was based on 5 trials, one from each of 5 original subjects for each SP of pitch change. Only 84 false-alarm distributions were derived, because SP has no meaning in terms of false alarms (the subjects' saying "different" when there were no pitch changes). False-alarm distributions were based on 25 trials (5 original subjects' responses to 5 trials in which there were no pitch changes) for each of the 84 conditions. This meant that five hit distributions for each of the 84 conditions were each paired with a single falsealarm distribution for each of the same 84 conditions in order to calculate the 420 MOC values for each supersubject.

The MOC values were subjected to a split-plot ANOVA in which musical experience ( 2 levels) was the betweengroups variable, and rhythmic frame (3 levels), center rhythms ( 7 levels), melodic rule (2 levels), type of pitchlevel accenting (2 levels), and SP of pitch change (5 levels) were repeated measures. Since the blocks within levels of musical experience were matched supersubjects, it was reasonable to assume that block and treatment effects were additive (Kirk, 1968). Therefore, criteria for reporting significant outcomes were relaxed to $p<.05$ (univariate $F$ test) and $p<.20$ (Geisser-Greenhouse $F$ test).

As can be seen from the first part of Table 7, all four main effects that were significant in the analysis of individual subjects' sensitivity were also significant in the supersubject analysis. The $F$ levels of all main effects increased due to reduced error variance, but degrees of freedom were also substantially reduced. Post hoc analysis revealed the same differences for the main effect of rhythmic frames as before: performance with amphibrach frames was significantly worse than performance with anapestic or dactylic frames, which did not differ. The main effect of center rhythms, which approached significance in the previous analysis $(p<.07)$ was found at approximately the same level of probability $(p<.10)$ in the supersubject analysis. The form of the rhythmic 
Table 7

Main Effects and Significant Interactions of Musical Variables on Supersubjects' Sensitivity to Pitch Change

\begin{tabular}{|c|c|c|c|c|}
\hline Variable & $F$ & $d f$ & $\begin{array}{c}\text { Univariate } \\
\text { Probability }\end{array}$ & $\begin{array}{c}\text { Geisser-Greenhouse } \\
\text { Probability } \\
\end{array}$ \\
\hline \multicolumn{5}{|c|}{ Effects to be Compared With Individual-Subjects Analysis } \\
\hline $\mathbf{E X}^{*}$ & 223.48 & 1,2 & .0044 & \\
\hline FR & 97.39 & 2,4 & .0004 & .0015 \\
\hline CR & 2.26 & 6,12 & .1076 & .2292 \\
\hline MR & 67.69 & 1,2 & .0145 & \\
\hline PLA & 22.10 & 1,2 & .0424 & \\
\hline $\mathrm{FR} \times \mathrm{PLA} \dagger$ & 2.68 & 2,4 & .1828 & .2406 \\
\hline \multicolumn{5}{|c|}{ Serial Position Effects and Interactions } \\
\hline SP & 15.62 & 4,8 & .0008 & .0325 \\
\hline $\mathbf{F R} \times \mathbf{S P}$ & 10.09 & 8,16 & .0001 & .0415 \\
\hline $\mathrm{CR} \times \mathrm{SP}$ & 3.97 & 24,48 & .00004 & .1209 \\
\hline PLA $\times$ SP & 7.90 & 4,8 & .0070 & .0848 \\
\hline $\mathrm{EX} \times \mathrm{FR} \times \mathrm{SP}$ & 3.38 & 8,16 & .0183 & .1600 \\
\hline MR $\times$ PLA $\times S P$ & 6.10 & 4,8 & .0149 & .0914 \\
\hline
\end{tabular}

Note-Dependent variable is area under the memory operating characteristic. Abbreviations: $\mathrm{EX}=$ experience, $\mathrm{FR}=$ rhythmic frame, $\mathrm{CR}$ = center rhythm, MR = melodic rule, PLA = type of pitch-level accenting, $\mathrm{SP}=$ serial position of pitch change. *Experience is a between-groups variable; other variables are repeated measures. †Nonsignificant interaction FR $\times$ PLA is included for the purpose of comparison with previous analysis of individual subjects' sensitivity.

frame $\times$ type of pitch-level accenting interaction was nearly identical to that found in the individual-subjects analysis, but had a reduced proportion of attributed variance and was not significant in the supersubject analysis. The interactions of rhythmic frame and type of pitch-level accenting with SP may account for part of this reduction.

Serial position (SP). The post hoc analysis of the significant main effect for SP showed that performance did not differ at SPs 3, 5, and 7, and that it was significantly better at these SPs than at SPs 4 or 6 (see Tables 7 and 8). We did not find, as did Jones et al. (1982), that performance was significantly worse at SP4 than at SP6. In fact, we found that discrimination was slightly but not significantly better at SP4 than at SP6. According to Jones et al. (1982), pitch recognition should be better at SP6 than at SP4 because a higher-order melodic rule is instantiated at SP4 and a lower-order rule is completed at SP6; therefore, pitch deviations should be easier to recognize at the latter SP than at the former. The main effect of SP is qualified by interactions with both rhythmic and pitch variables that may help to explain the differences in results.

Rhythm variables, musical experience, and serial position. The interaction of rhythmic frame with SP for the two musical-experience groups is shown in Figures 9A and $9 \mathrm{~B}$. According to the beacon hypothesis, we should expect best performance at SP3 with anapestic frames and at SP7 with dactylic frames. According to the nonconsonance hypothesis, we should expect, at all serial positions, a general advantage for dactyl and anapest frames, because they set up a clock that is consonant with pitchlevel accenting, and a general disadvantage for am- phibrach frames, which tend to set up out-of-phase consonance or dissonance with pitch-level accenting. Post hoc analysis showed that the effect of rhythmic frame was significant at SPs 3, 4, and 7. Since no frame $\times$ center rhythm interaction occurred, there is little evidence that the effect of timing of the frame extended its influence to recognition of tones outside the frame itself, except at SP4. For SP3, the overall order of performance, from best to worst, was anapestic, dactylic, amphibrach; post hoc analysis showed differences among all means to be significant. For SPs 4 and 7, the overall order of performance, from best to worst, was dactylic, anapestic, amphibrach; for SP4 there was a significant difference between amphibrach and both dactylic and anapestic frames, which did not differ; for SP7, the only significant difference occurred between dactyl and amphibrach frames. These findings are congruent with both the Beacon hypothesis and the nonconsonance hypothesis.

The significant three-way interaction of experience, rhythmic frame, and SP further qualifies the above interaction and indicates that musicians and nonmusicans reacted differently to the effect of rhythmic frames at different SPs. The two groups performed similarly at SPs 5,6 , and 7. However, at SP3, although the ordering of frame means was the same for the two groups, the only significant difference for musicians was between anapest and amphibrach (representing a 6\% difference in area under the MOC); for nonmusicians, all differences among frame means were significant, with the largest representing an $11 \%$ difference in area under the MOC. From this it is clear that nonmusicians' performance is more highly disrupted at SP3 by the amphibrach frame. At SP4, musicians performed significantly better with dactylic and anapestic than with amphibrach frames; nonmusicians performed equally poorly with all frames. Therefore, it is the musicians' performance that contributes most to any carryover effect of frame to SPs that are primarily governed by center rhythms. This result lends credence to the notion that temporal regularity of dactyl and anapest frames at the lowest hierarchical level sets up an internal clock among musicians that leads to better pitch perception at the next tick of that clock-namely, at SP4.

Center rhythms and serial position. Post hoc analysis showed that the interaction of center rhythms with SP occurred only at SPs 4 and 6. This interaction will be described in two parts. First, center rhythms with filled intervals $(211,112$, and 121) show a pattern similar to

Table 8

Supersubjects' Sensitivity to Pitch Change as a Function of Serial Position of Change for Levels of Musical Experience

\begin{tabular}{lcccccc}
\hline & \multicolumn{6}{c}{ Serial Position of Pitch Change } \\
\cline { 2 - 7 } Experience & 3 & 4 & 5 & 6 & 7 & Mean \\
\hline Musicians & .957 & .896 & .977 & .907 & .956 & .939 \\
Nonmusicians & .869 & .821 & .917 & .805 & .862 & .855 \\
Mean & .913 & .859 & .947 & .856 & .909 & \\
\hline
\end{tabular}

Note-Dependent variable is area under the memory operating characteristic; chance performance is .50 . 

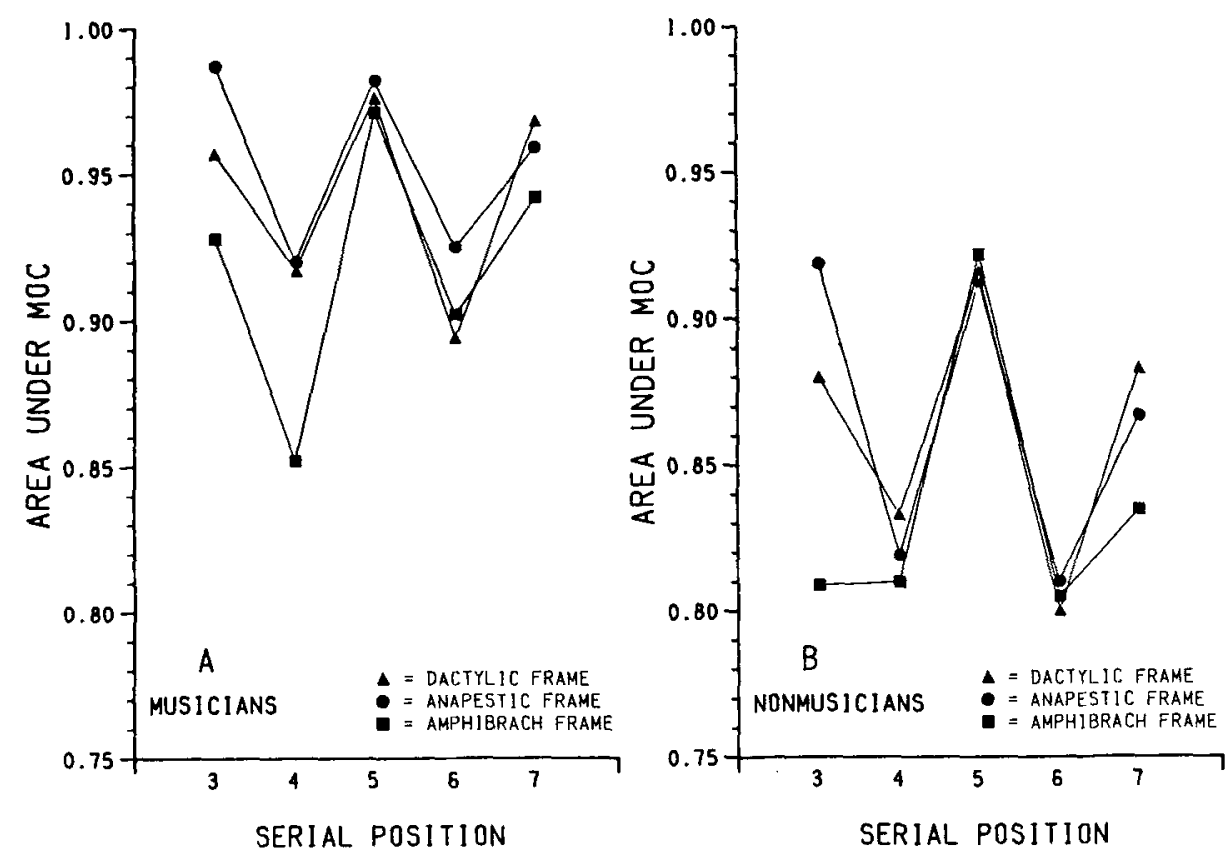

Figure 9. Supersubjects" sensitivity to pitch differences as a function of serial position of pitch change for different degrees of musical experience and kinds of rhythmic frame. Panel $A$ shows the average sensitivity of 2 musician supersubjects; Panel B shows the average sensitivity of 2 nonmusician supersubjects. The measure of sensitivity is area under the memory operating characteristic (MOC), where chance performance is .50 .

that discovered for rhythmic frames (see Figure 10A). SP4 shows an advantage for dactylic center rhythm and SP6 shows an advantage for anapestic center rhythm. The poorest performance is associated with the amphibrach center rhythm (121), except for SP5, where all center rhythms are associated with excellent recognition performance. There is no evidence that center rhythms have any influence on pitch recognition performance beyond the tones they govern, because means for center rhythms do not differ at SPs 3 and 7 and there was no significant interaction of center rhythms with rhythmic frames.

Second, center rhythms that include rests show relatively poor performance at SPs 4 and 6 for the restamphibrach pattern (11R1), and no particular advantage for any of the other patterns (1R11, 111R, and R111), all of which evoked very similar performance (see Figure 10B). Comparison of Figures 10A and 10B suggests that anapestic and dactylic filled-interval patterns (211 and 112) usually promoted somewhat better performance at SPs 4 and 6, respectively, than did their rest pattern counterparts (1R11 and 111R). Also, the filledinterval amphibrach pattern 121 was far less disruptive to pitch recognition than was its rest pattern counterpart (11R1), especially at SP4. Post hoc analyses showed that the only significant differences among center rhythms at SP4 were between the rest-amphibrach rhythm (11R1) and the filled-interval dactylic, anapestic, and amphibrach center rhythms $(211,112$, and 121). At SP6, both 121 and 11R1 rhythms differed from anapestic rhythms, whereas the $11 R 1$ rhythm differed from all other rhythms.

These results again suggest the disruptiveness of the amphibrach rhythm to pitch recognition; they also suggest that, relative to filled intervals, empty intervals or rests may be less clear in their rhythmic interpretation and generally less helpful to listeners (especially to nonmusicians) in their attempts to remember the pitches of a melody.

Pitch variables and serial position. Figure 11 shows the MOC values of the four melodic patterns when pitch changes occur at each of five SPs. There was a significant type of pitch-level accenting $\times$ SP interaction (open vs. filled markers in Figure 11), which conferred a pitchdiscrimination advantage to early-inflected over earlypitch-skip melodies at SPs 3, 4, and 6. The unique effects for the one-rule early-inflected melody (Melody 2) are evident in the significant three-way interaction of melodic rule, inflection point, and SP that occurs at SP6. The effects at SPs 3 and 6 are due entirely to the fact that comparisons for early-inflected Melodies 2 and 4 changed the pitch contour of the standard; also note that the effect at SP6 must have also occurred in Jones et al.'s (1982) experiment. Therefore, any unconfounded advantage of the one-rule repetition patterns or of early-inflection melodies occurred at SP4. Post hoc analysis showed that oneand two-rule early-inflected melodies differed from the two-rule early-pitch-skip melody at SP4. We interpret this 

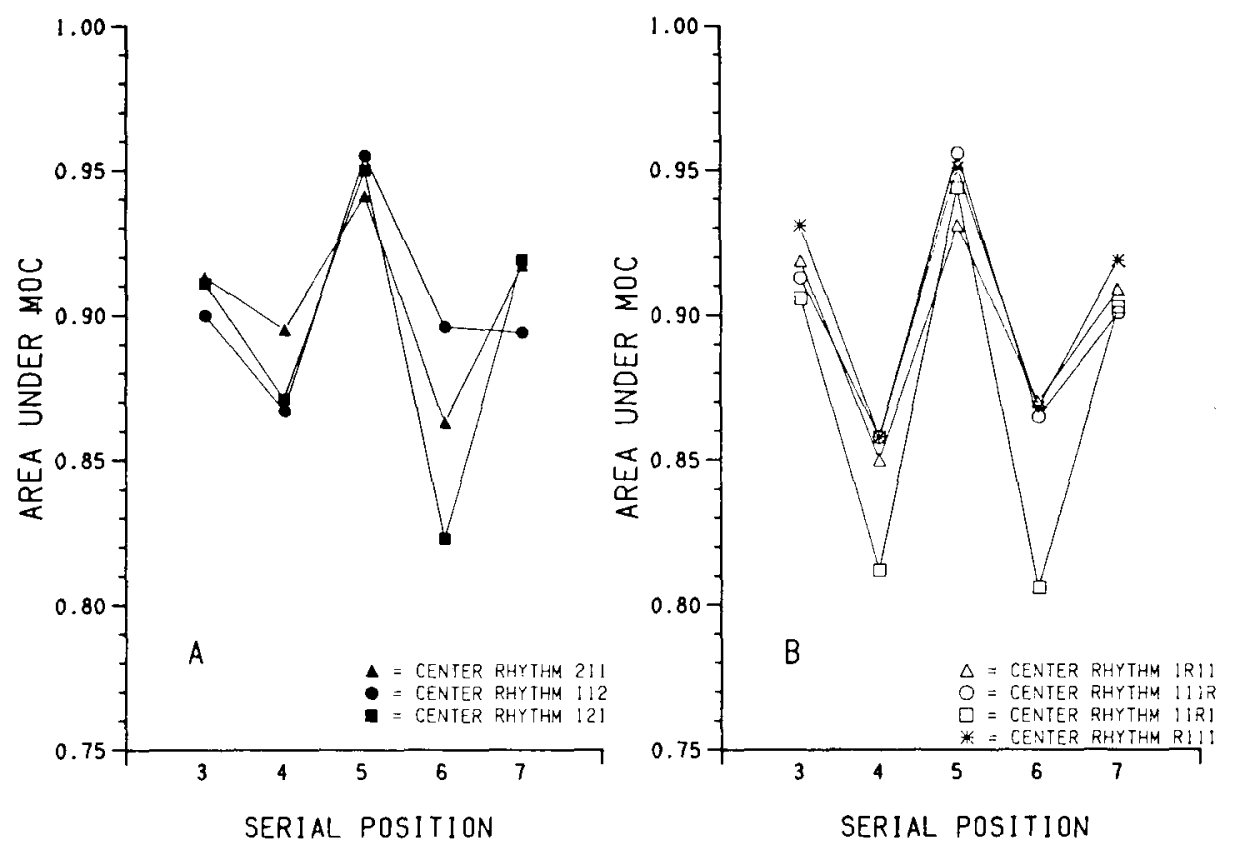

Figure 10. Supersubjects' sensitivity to pitch differences as a function of serial position of the pitch change for different center-phrase rhythms. Panel A shows sensitivity for center-phrase rhythms in which a lengthened tone marks accents $(211,112$, and 121); Panel B shows sensitivity for center-phrase rhythms that include rests (1R11, 111R, 11R1, and R111). The measure of sensitivity is area under the memory operating characteristic (MOC), where chance performance is . $\mathbf{5 0}$.

finding to mean that a contour inflection makes chroma changes at SP4 more salient than does a unidirectional pitch skip. Repetition of the first 3 tones at SPs 7, 8, and 9 in the one-rule early-inflected melody may have helped discrimination, because the final tone of this melody would provide the same reference for a changed pitch as would the third tone just preceding SP4.

Pitch variables may account for our results as follows: (1) Pitch changes at SP3 always involved deviations from the same pitch in all four melodies, and in the case of early-inflected melodies always changed the standard melody's contour. (2) Generally low recognition scores at SP4 may have been due to the instantiation of a higherorder pitch rule, but a higher-order pitch rule was also instantiated at SP7, which had relatively high recognition scores. (3) Performance was poorer at SP4 for melodies with pitch-level accenting in the form of a unidirectional pitch skip as opposed to a pitch contour inflection (between SPs 4 and 5). (4) One-rule patterns tended to be easier than two-rule patterns at SP4. (5) Changes at SP5 always entailed a change in pitch contour from the standard pattern.

It is very difficult to account for the generally poor performance at SP6 and the good performance at SP7 on the basis of the pitch variables we tested. We note that, in addition to the possible explanations in terms of pitch factors, the generally good performance at SPs 3 and 7 may be accounted for by the fact that they were always part of a rhythmic frame and were subject to many fewer tem- poral variations and comprised more long filled intervals than did SPs 4, 5, and 6, which were always part of a center rhythm. With the exception of relatively poor performance for early-pitch-skip and two-rule melodies at SP4, the generally poor performance at SPs 4 and 6 may be accounted for by temporal factors. The outstanding performance at SP5 is accounted for by the fact that all deviant comparisons changed the pitch contour of the standard.

\section{The Nonconsonance Hypothesis as an Explanation of Results}

Thus far we have concentrated on results at a rather molecular level. Table 9 shows musicians' and nonmusicians' pitch-difference discrimination performance for each of the 21 rhythms. The patterns are placed in categories determined by our nonconsonance hypothesis; patterns are ranked according to performance within these categories. Most category weights for pattern difficulty are 2 or 0 : these represent the predicted number of bestclock temporal accents in each pattern that coincide with pitch-level accenting at SPs 4 and 7. There is one major exception to this rule: We gave consonant patterns with disruptive (121 or 11R1) center rhythms a weight of 1 because we felt listeners might reset their internal clocks, since the points at which they should "tick"- that is, at the first or third interval of these center rhythms-have no natural accent or no note onset, respectively. It was also a bit difficult to know exactly where to place the $R+3 / 8$ (R111 center rhythm) patterns; we have put the 


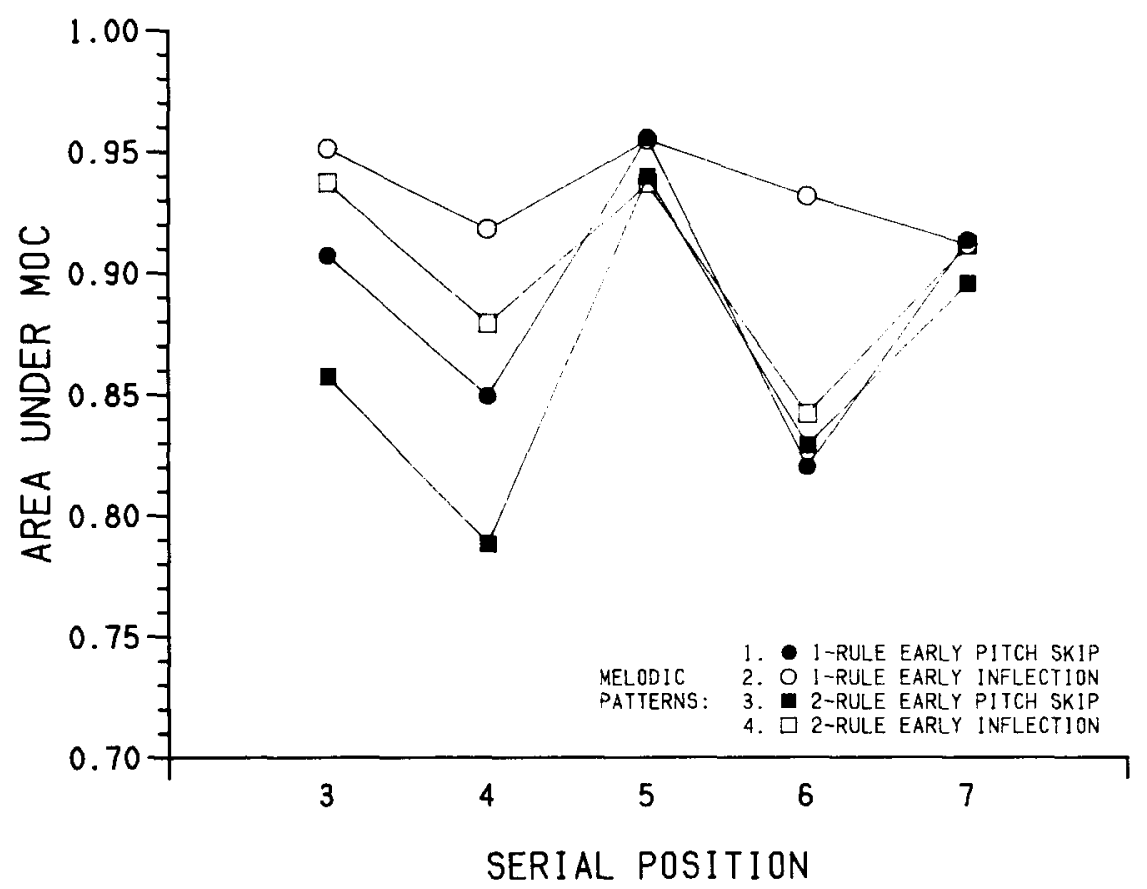

Figure 11. Supersubjects' sensitivity to pitch differences as a function of serial position of the pitch change for different types of melody. There were four types of melody: one-rule and two-rule, each of which had two kinds of pitch-level accenting in the form of an inflection or an unusual unidirectional pitch skip early in the pattern. The measure of sensitivity is area under the memory operating characteristic (MOC), where chance performance is .50.

dactyl and anapest frame patterns with these center rhythms (211 R111 211 and 112 R111 112) in the consonant category.

We placed the $121 \mathrm{R} 111121$ pattern (best-clock accents are underlined) in the out-of-phase category, because listeners clearly hear this pattern with a meter of base 2 starting with the second note. Since the pitch-level accent at SP4 follows a rest in this pattern, the best-clock temporal accent is in phase with pitch-level accenting at SP4 and out of phase with it at SP7. This is the only pattern for which we chose a difficulty level that was not congruent with the category of the pattern.

Linear regression of mean group performance on the 21 patterns was fitted to these category weights for musicians and nonmusicans and for their average: $R^{2}(19)=$ $.633, .421$, and .574 , respectively, $p<.001$ for all regressions. These categories account for well over half the variance in musicians' and overall average performance; therefore, we view this as a substantial confirmation of our nonconsonance hypothesis.

The correlation of musicians' and nonmusicans' performance on the 21 patterns was $.467, p<.05$, $R^{2}=.218$. Therefore, although it appears that musicians and nonmusicans respond to some of the same variables, there are enough differences in performance to warrant different musical grammars for their responses to rhythm and metricality; one major roadblock to a grammar for nonmusicians is the fact that their behavior is much more variable than that of musicians.

\section{Generality of Results}

Musicians showed a very slight tendency for better performance with anapestic than with dactylic frames (five of the six best pattern performances for musicians had anapestic frames). Nonmusicians showed no such tendency (see Table 9; also see Figures 9A and 9B). The grouping of the pitch patterns is entirely clear in the initial part of the 112 frame because grouping by the proximity of note onsets perfectly matches grouping by the proximity of pitch levels: C4D4E4.G4A5B5..., instead of C4.D4E4G4.A5B5... The two short notes of the dactyl tend to become upbeats to the following pitch-level accent, and the pitch rule C4D4E4 becomes more difficult to hear. Performance may suffer because of this. An additional weighting favoring anapestic frames would help us to predict musicians' performance.

Nonmusicians' performance seems to be driven by the attentional beacon of the long filled interval, or, equivalently, by an inability to ignore temporal accenting and grouping in favor of pitch-level accenting and grouping (provided by the frequency contiguity of pitches following the simple +1 of -1 pitch rule). Our supposition is that a model that incorporates an expectancy of regularity of temporal contour, a regularity in the series of longs and shorts of a pattern, will fit nonmusicians' performance better than a model that considers only metrical regularity (like the one we have presented). Povel and Essens (1985, Experiment 2) have made a start on a temporal contour or rhythm coding model; how such a model 
Table 9

Musicians' and Nonmusicians' Pitch-Difference Detection Performance as a Product of Entire Rhythm Patterns

\begin{tabular}{|c|c|c|c|c|}
\hline \multirow[b]{2}{*}{ Rhythm Pattern } & \multirow[b]{2}{*}{ Difficulty* } & \multicolumn{3}{|c|}{ Area Under the MOC' } \\
\hline & & Musicians & Nonmusicians & Mean \\
\hline \multicolumn{5}{|c|}{ Consonant Patterns } \\
\hline 1. $112\|1\| \mathrm{R} \quad 112$ & 2 & .973 & .885 & .939 \\
\hline 2. 211112211 & 2 & .945 & .905 & .925 \\
\hline 3. 211211211 & 2 & .938 & .902 & .920 \\
\hline 4. $112 \mathrm{R} 111112$ & 2 & .947 & .881 & .914 \\
\hline 5. 112211112 & 2 & .953 & .872 & .913 \\
\hline 6. 112112112 & 2 & .964 & .858 & .911 \\
\hline 7. $|\|2 \quad \mid R\| 1 \| 12$ & 2 & .957 & .865 & .911 \\
\hline 8. $211 \mathrm{R} 11 \| 211$ & 2 & .957 & .855 & .906 \\
\hline 9. 21$\} 1 R 11211$ & 2 & .929 & .865 & .897 \\
\hline $10.211111 \mathrm{R} 211$ & 2 & .951 & .830 & .891 \\
\hline
\end{tabular}

Consonant Patterns With Disruptive Center Rhythms

$\begin{array}{lllll}11.211121211 & 1 & .941 & .872 & .906 \\ 12.112121112 & 1 & .933 & .861 & .897 \\ 13.11211 \mathrm{R} 1112 & 1 & .955 & .837 & .896 \\ 14.211 \mathrm{IR} 1211 & 1 & .934 & .807 & .870\end{array}$

Out-of-Phase Consonant Patterns

$\begin{array}{lllll}\text { 15. } 121 \mathrm{R} 111121 & 1 & .937 & .855 & .896 \\ 16.121121121 & 0 & .925 & .838 & .881 \\ 17.121 \mathrm{IIRI} 121 & 0 & .894 & .817 & .817\end{array}$

\begin{tabular}{lllll} 
18. I21 211 121 & 0 & .917 & .853 & .885 \\
$19.1211 \mathrm{R} 11121$ & 0 & .918 & .840 & .879 \\
$20.121111 \mathrm{R} 121$ & 0 & .929 & .821 & .875 \\
21.121112121 & 0 & .913 & .829 & .871 \\
\hline
\end{tabular}

Note-MOC = memory operating characteristic. *Predicted difficulty is a function of pitch-level accenting coinciding with the temporal best clock ( 2 = easy, 1 = moderately difficult, $0=$ difficult). $\quad+$ Chance performance is .5 .

should be fit with pitch pattern coding is a topic for future investigation.

An example of how nonmusicians' performance seems to be affected by local temporal accenting is in their performance with the 121211121 nonconsonant pattern. If nonmusicians were able to impose a metric at all, they probably grouped this pattern in metric base 3 , since it starts with a repeated [12] temporal grouping (i.e., 1212 11121 ). Their area under the MOC was .777 at SP3 and a relatively high .825 at SP4. Musicians' area under the MOC for the same pattern was .925 at SP3 and .899 at SP4; this suggests to us that musicians' performance is dictated not so much by local temporal accents, but more by their attempts to impose a metric that will fit with the regularity of accents in the pitch pattern. In fact, most of the musicians, after hearing these patterns over and over again, were probably able to hear the regularity of the +1 or -1 pitch rule; that is, they could clearly hear a metric in base 4 . This is surely the case with the relatively difficult out-of-phase consonant pattern 121121 121; one can impose a perceptual clock that is consonant with the pitch-level accenting (in metric base 4 , starting on the first note), but to do so one must ignore three longinterval temporal accents that are out of phase with pitch- level accenting. The division of attention between temporal and pitch-level accenting clearly results in a decrement in pitch-difference discrimination performance for both musicians and nonmusicians. The decrement is greater among nonmusicians because they do not seem to appreciate the ratios among temporal intervals and are apparently less able to generate longer metrics (e.g., in base 4).

\section{Observations About Patterns \\ That Include Rests}

The only generalization one can make about the center rhythms that include rests is that the amphibrach-rest pattern $(1 \mid R I)$ is the most disruptive to pitch-difference discrimination performance (at SPs 4 and 6) and is generally slightly more disruptive than its filled-interval counterpart (121) in the context of the same frame. But even this rule has an exception: musicians' area under the MOC with this center rhythm was .955 in the context of an anapest frame (112 11R1 112), and .933 with 112121 112 (cf. patterns 12 and 13, Table 9). In short, the helpfulness or disruptiveness of a partially filled interval depends in complex ways on context and listeners' experience.

We planned to compare the patterns $211 \mathrm{R} 111211,211$ 211211 , and $2111 \mathrm{R} 11211$ to see which center rhythm provided the best cue for accenting and pitch-difference discrimination. Performance differences at SP4 were not significant for these patterns; however, the overall order of performance, from best to poorest, indicates $211>\mathrm{R} 111>1 \mathrm{R} 11$. Therefore, the long filled interval appears to provide the best pitch-discrimination cue.

\section{Comparison With Results \\ of a Previous Experiment}

Table 10 shows a comparison of four conditions in the present experiment with four similar conditions in Jones et al.'s (1982) experiment. The measures, as they stand, are not directly comparable, because Jones et al. used the probability of correct detection of a pitch difference: this would be equivalent to our hit rate measures for each condition, which we do not report because of the difficulty of dividing a 7-point scale into right and wrong answers.

Table 10

Comparison of Four Conditions in Two Experiments

\begin{tabular}{lccc}
\hline & SP4 & SP6 & Mean \\
\hline Area Under the MOC in the Present Experiment & \\
AUU Context & .885 & .849 & .862 \\
UUA Context & .911 & .910 & .911 \\
Mean & .898 & .879 &
\end{tabular}

Probability of Correct Detection of a Pitch Change in Jones, Boltz, \& Kidd (1982)

\begin{tabular}{llll} 
AUU Context & .740 & .807 & .778 \\
UUA Context & .583 & .880 & .732 \\
Mean & .662 & .843 & \\
\hline
\end{tabular}

Note-MOC = memory operating characteristic; $S P=$ serial position. 
However, since Jones et al. reported almost identical false-alarm rates (the probability of saying there was a pitch difference between patterns when the patterns were the same in pitch) for all their conditions, an area-underthe-MOC measure in their experiment would be almost solely a function of the hit rates. So at least ordinal comparisons are possible, and they are indicative of two major differences in outcome.

Jones et al. (1982) showed a distinct advantage for detection of pitch differences at SP6 over SP4, and a slight advantage of the $211 \mathrm{R} 111211$ pattern (which they called "AUU context") over the 112 111R 112 pattern (which they called "UUA context"). Our results showed a distinct advantage for the so-called UUA context over the AUU context [in a planned comparison, $t(3)=5.65$, $p<.01]$ and no difference between SPs 4 and 6 .

One possible reason for this difference is that in Jones et al.'s (1982) experiment, rhythm was a between-subjects variable, and in the present study, it was a within-subjects variable. Musical experience accounted for the major portion of variance in the present experiment. Jones et al. did not measure musical experience and assumed they had controlled for this factor by random assignment of subjects to conditions ( $n=6$ per condition). So there is the possibility of subject selection for experience in Jones et al.'s results. Another possibility is that all of Jones et al.'s subjects were relatively untrained musically, so that the major cue they responded to at SP6 was the obvious contour break that must occur there when a one-rule early-inflected melody is played with changed pitch. This would amount to an experience $\times$ pitch pattern $\times$ rhythm interaction. Musical experience does not account for our results. Performance averaged over the levels of experience (see Table 10) is representative of similar patterns of response by musicians and nonmusicians. This can be partly confirmed by comparing performance for Pattern 1 (AAU context) and Pattern 8 (UUA context) for the two groups in Table 9.

Jones et al.'s (1982) listeners heard different pitch patterns with a single rhythm as the standard, 80 different times; by contrast, each of our listeners heard each of 21 rhythms as the standard 40 different times. Even though Jones et al. included dummy trials so that subjects would not always listen for changes at a particular SP, changes at SPs 4 and 6 were relatively more common for their subjects than for ours ( $15 \%$ of total trials each vs. $10 \%$ ). Therefore, it may be that our results reflect what listeners do after hearing many different rhythm and pitch combinations with a relatively unpredictable point of pitch deviation, whereas Jones et al.'s reflect what listeners do after a large amount of practice with a single rhythm with changes made at the same SPs.

Also, because our subjects heard many more rhythm types than did Jones et al.'s subjects, they may have begun to develop a general metrical strategy for dealing with all rhythms, instead of just one. We interpret the significant difference that we found between the so-called AUU and UUA contexts for SPs 4 and 6 as a difference in the difficulty of dealing with a somewhat ametrical and an extremely metrical sequence, respectively. A best clock in Povel and Essens's (1985) sense does not line up pitchlevel and temporal accents for the AUU context, but it does for the UUA context. Also, as we have noted, grouping by the Gestalt principles of pitch contiguity and temporal contiguity is perfectly aligned in all-anapestic patterns, which is not the case for dactylic patterns.

We previously asked why it was that SP6, which has enormous predictability as to its pitch (the +1 rule is followed here), showed such poor pitch-discrimination performance; on the other hand, why did SP7, which has enormous unpredictability as to its pitch (in three of four melodies it is preceded by a large pitch skip ) show relatively good performance? We suggested that the reasons lay with the variability of the timing of the onsets of tones subsumed under each SP and the likelihood of occurrence of a longer sound-filled interval at each SP.

Another explanation, which we are not prepared to explore at length here, is that the pitch changes at SP6 all involved deviations from B4 (Melodies 1 and 3) or D4 (Melodies 2 and 4). Listeners may not have as good a tonal memory for these as for the highly tonal $\mathrm{C} 4$ (Melodies 1 and 2), F4 (Melody 3), and G4 (Melody 4) that occur at SP7. Comparison changes at SP7 might be easier to detect, since they involve going from tonal to less tonal pitches; comparison changes at SP6 might be harder, since they involve going from less tonal to more tonal pitches. Recall that we did not control for the tonality of our pitch changes in the same way that Jones et al. (1982) did. Dowling and Bartlett (1981) reported that listeners have a great deal of difficulty in detecting differences between same-contour patterns if the comparison pattern's intervals reinforce the tonality or key of the standard pattern; Dowling and Bartlett called these patterns "tonal lures." So what we may have done was to create tonal lures as comparisons in the case of SP6 and relatively atonal lures in the case of SP7.

This alternative explanation may also partially explain why we found no differences between the levels of performance at SP4 and SP6. Changes at SP4 were always from F4 to G4 or vice versa; they had to take these values, otherwise the comparison would have a different contour than the standard. So if the changes we made at SP4 and SP6 both reinforce the $\mathrm{C}$ major tonality, they should both be equally difficult to detect, as we found. If Jones et al. made the same changes that we did at SP4 in order not to break the contour of the standard, and if they avoided the highly tonal $\mathrm{C} 4, \mathrm{C} 5$, or $\mathrm{G} 4$ as changes at SP6, then it should have been easier for their listeners to detect a difference at SP6 than at SP4, which is what they found.

Although the above alternative may partly explain the level of results across the various SPs, it does not explain the patterning of results within each SP, which we interpret as a function of the long filled-interval beacon hypothesis and the nonconsonance hypothesis. 


\section{GENERAL OBSERVATIONS AND CONCLUSIONS}

Research psychomusicologists are usually on the horns of a dilemma. On the one hand, we are aware that the many carefully chosen and exactingly manipulated stimuli we employ are not "real music." On the other hand, we know that there are usually too many variables in performed music to attribute variance reliably to any one cause. So we have attempted a middle course, which is probably not totally satisfying to either musicians or psychologists.

By the criteria of psychologists and psychophysicists, we fail because we have not controlled or varied certain factors (most notably, in the present study, tonality and tempo). Also, interactions are difficult to deal with, especially in regard to musical experience; in choosing a subject population we are often making a choice between what people can do (musicians) and what most people usually do (nonmusicians), and the latter population is notoriously more variable than the former.

By the criteria of musicians, we fail because we have left out the interpreter of the notes on paper-the performer. As an example of this, we have pointed out in our paper how unlikely it is that listeners will hear either a dactyl or amphibrach pattern based on the fact that we have varied only the IOI between tones; the pitch patterns one hears under such circumstances are usually grouped by the temporal contiguity of note onsets. A live performer would surely be able to make these patterns sound like combinations of dactyls and amphibrachs, but in doing so he/she would be varying loudness, attack pressure, durations of tones within the prescribed interval, tempo, and perhaps other variables; and he/she would accomplish this in ways that might differ from those of other performers. As it stands, even the tones in our stimuli show small differences in time-variant amplitude. In future projects we intend to compare data for live-performance stimuli with those for computer-generated patterns.

For the way we ran our experiment, the only patterns for which we are certain that listeners heard three separate 3 -tone motifs, each composed of pitches related by +1 or -1 diatonic scale steps, are those with temporal bars made up of anapests. The Gestalt principles of grouping by temporal contiguity of note onsets and frequency contiguity of pitches make it very difficult to impose other groupings. Pitch-difference discrimination was best for musicians with this type of temporal patterning. We note that such patterning also marks some of our bestremembered folk songs, such as "Three Blind Mice." Strong pitch level and weak temporal accenting begin each phrase and strong temporal accenting ends it. The next question we intend to address is: What kinds of temporal variation permit the listener to report that he hears a similar melody? The question we asked in the present experiment was the opposite side of the coin: What kinds of temporal variation make it difficult to hear a single pitch chroma difference between two melodies that are other- wise identical? We feel comfortable in making the following conclusions:

1. Musical experience accounted for nearly half the variance in our results. Musicians performed better than nonmusicians on every pattern we tested.

2. Pitch patterning accounted for some results at particular SPs: a pitch-level accent in the form of a pitch inflection instead of a unidirectional pitch skip resulted in better performance at SP4. Patterns in which the initial 3 -tone motif was repeated in the last 3 tones (one-rule patterns) resulted in better performance at SP4. We note also that some comparisons always changed the standard's contour at SPs 3, 5, and 6, and this resulted in better performance for those patterns. Musicians' and nonmusicians' performance did not significantly differ as a product of the pitch variables we tested.

3. Three major relationships between temporal accenting and pitch-level accenting were explored: (1) Rhythmic consonance, the case where there is perfect alignment or nesting of temporal accenting by pitch-level accenting, resulted in the best pitch-difference discrimination, especially when temporal accenting was in the form of a long filled interval. (2) Out-of-phase consonance and (3) dissonance are structurally different cases where temporal and pitch-level accenting either are nested differently or occur at different rates. Both of these cases result in internal clocks or levels of motion that are in conflict with one another, resulting in a perception of syncopation and poorer pitch-difference discrimination for these patterns.

4. There is some evidence that musicians were able to use the regular metrical information in the early portion of rhythmically consonant patterns to help them detect pitch differences better at SP4.

5. Musicians and nonmusicians performed sufficiently differently on the patterns we tested (sharing only $21 \%$ of the total variance) to warrant attempts to find different bases for their behavior. Generally, nonmusicians' performance seemed to be influenced less by a metrical perception of the patterns than by the temporal accenting provided by the position of tones initiating long filled intervals.

We are struck by the parallel between tonality and metricality as they relate to the performance of musicians and nonmusicians. Musicians use hierarchical tonal and metrical schemata to organize musical patterns. In the pitch realm, nonmusicians seem to be most influenced by considerations of pitch height and not by the complex hierarchical and ratio relations among pitches that a learned tonal system engenders (Krumhansl \& Kessler, 1982). In the temporal realm, nonmusicians are influenced by the direction of size differences (that is, temporal contour, longer or shorter) between successive temporal intervals, but have little appreciation for the ratios between temporal intervals and how these might be nested within longer hierarchically related intervals.

We suggest that our findings indicate that composers who ignore the Gestalt principles of grouping, in the do- 
main either of frequency (pitch) or of time, run the risk of writing music that is difficult to code or to remember easily in the short term. For long-term memory, it is an empirical question whether the pitches of a melody with consonant accenting would be better remembered than those with nonconsonant accenting. Both musicians and nonmusicians, but especially the latter, seem to have definite processing limitations that do not easily permit them to divide their attention between events that occur at different rates or are out of phase with each other. Different training may allow some listeners to keep multiple or compound internal clocks, as has been suggested from evidence on African drum ensembles (Locke, 1982); but that is not the training of a typical Western youngster.

\section{REFERENCES}

Boltz, M., \& Jones, M. R. (1986). Does rule recursion make melodies easier to reproduce? If not, what does? Cognitive Psychology, 18, 389-431.

Bregman, A. S., \& Campbell, J. (1971). Primary auditory stream segregation and perception of order in rapid sequences of tones. Journal of Experimental Psychology, 89, 244-249.

CicchetT, D. V. (1972). Extensions of multiple-range tests to interaction tables in the analysis of variance: A rapid approximate solution. Psychological Bulletin, 77, 405-408.

Clarke, F. R. (1964). Confidence ratings, second-choice responses, and confusion matrices in intelligibility tests. In J. A. Swets (Ed.), Signal detection and recognition in human observers. New York: Wiley.

Collard, R., \& Povel, D.-J. (1982). Theory of serial pattern production: Tree reversals. Psychological Review, 89, 693-707.

COOPER, G. \& MEYER, L. B. (1960). The rhythmic structure of music. Chicago: University of Chicago Press.

Crowder, R. G. (1982). Decay of auditory memory in vowel discrimination. Journal of Experimental Psychology: Learning, Memory \& Cognition, 8, 153-162.

DEuTSCH, D. (1980). The processing of structured and unstructured tonal sequences. Perception \& Psychophysics, 28, 381-389.

DEUTSCH, D., \& FEROE, J. (1981). The internal representation of pitch sequences in tonal music. Psychological Review, 88, 502-522.

Dowling, W. J. (1968). Rhythmic fission and perceptual organization of tone sequences. Unpublished doctoral dissertation, Harvard University, Cambridge, MA.

Dowling, W. J. (1971). Recognition of inversions of melodies and melodic contours. Perception \& Psychophysics, 9, 348-349.

Dowling, W. J. (1972). Recognition of melodic transformations: Inversion, retrograde and retrograde inversion. Perception \& Psychophysics, 12, 417-421.

Dowling, W. J. (1973). The perception of interleaved melodies. Cognitive Psychology, 5, 322-337.

Dowling, W. J., \& BARTLETT, J. C. (1981). The importance of interval information in long-term memory for melodies. Psychomusicology, 1, 30-49.

Dowling, W. J., \& FujITANI, D. S. (1971). Contour, interval and pitch recognition in memory for melodies. Journal of the Acoustical Society of America, 49, 524-531.

Dowi ing, W. J., \& Harwood, D. L. (1986). Music cognition. Orlando, FL: Academic Press.

GABRIELSSON, A. (1985). Interplay between analysis and synthesis in studies of music performance and music experience. Music Perception, 3, 59-86.

GARNER, W. R. (1974). The processing of information and structure. Potomac, MD: Erlbaum.

Geisser, S., \& GreENhouse, S. W. (1958). An extension of Box's results on the use of the F distribution in multivariate analysis. Annals of Mathematical Statistics, 29, 885-891.
Handel, S., \& Lawson, G. R. (1983). The contextual nature of rhythmic interpretation. Perception \& Psychophysics, 34, 103-120.

HANDEL, S., \& OSHINSKY, J. S. (1981). The meter of syncopated auditory polyrhythms. Perception \& Psychophysics, 30, 1-9.

JONES, M. R. (1974). Cognitive representations of serial patterns. In B. Kantowitz (Ed.), Human information processing: Tutorials in performance and cognition. Potomac, MD: Erlbaum.

JONES, M. R. (1976). Time, our lost dimension: Toward a new theory of perception, attention, and memory. Psychological Review, 83, 323-355

Jones, M. R. (1978). Auditory patterns: The perceiving organism. In E. C. Carterette \& M. P. Friedman (Eds.), Handbook of perception: Vol. 8. Perceptual coding. New York: Academic Press.

JoNEs, M. R. (1981). A tutorial on some issues and methods in serial pattern research. Perception \& Psychophysics, 30, 492-504.

Jones, M. R., Boltz, M., \& KIDD, G. R. (1982). Controlled attending as a function of melodic and temporal context. Perception \& Psychophysics, 32, 211-218.

Jones, M. R., MASER, D. J., \& KIDD, G. R. (1978). Rate and structure in memory for auditory patterns. Memory \& Cognition, 6, 246-258.

Kirk, R.E. (1968). Experimental design: Procedures for the behavioral sciences. Belmont, CA: Brooks/Cole.

Krumhansl, C. L., \& Kessler, E. J. (1982). Tracing the dynamic changes in perceived tonal organization in a spatial represenation of musical keys. Psychological Review, 89, 334-368.

Linton, M., \& Gallo, P. S. (1975). The practical statistician. Belmont, CA: Wadsworth.

LOCKE, D. (1982). Principles of offbeat timing and cross-rhythm on southern Eve dance drumming. Ethnomusicology, 26, 217-246.

Longuet-Higgins, H. C., \& LEE, C. S. (1982). The perception of musical rhythms. Perception, 11, 115-128.

McNicoL, D. (1972). A primer of signal detection theory. London: Allen \& Unwin.

Monahan, C. B. (1984). Parallels between pitch and time: The determinants of musical space. Dissertation Abstracts International, 45, 1942B. (University Microfilms No. 84-20, 214)

Monahan, C. B., \& Carterette, E. C. (1985). Pitch and duration as determinants of musical space. Music Perception, 3, 1-32.

Povel, D.-J. (1981). Interval representation of simple temporal patterns. Journal of Experimental Psychology: Human Perception \& Performance, 7, 3-18.

PoVEL, D.-J. (1984). A theoretical framework for thythm perception. Psychological Research, 45, 315-337.

Povel, D.-J., \& Essens, P. (1985). Perception of temporal patterns. Music Perception, 2, 411-440.

Povel, D.-J., \& OKKerman, H. (1981). Accents in equitone sequences. Perception \& Psychophysics, 30, 565-572.

RESTLE, F. (1970). Theory of serial pattern learning: Structural trees. Psychological Review, 77, 481-495.

SAmUEL, A. G. (1981). Phonemic restoration: Insights from a new methodology. Journal of Experimental Psychology: General, 110, 474-494.

SCHOLES, P. A. (1964). The concise Oxford dictionary of music. London: Oxford University Press.

Simon, H. A., \& Sumner, R. K. (1968). Pattern in music. In B. Kleinmuntz (Ed.), Formal representation of human judgment. New York: Wiley.

Steedman, M. J. (1977). The perception of musical rhythm and metre. Perception, 6, 555-570.

THOMASSEN, J. M. (1982). Melodic accent: Experiments and a tentative model. Journal of the Acoustical Society of America, 71, 1596-1605.

VAN NoORDEN, L. P. A. S. (1975). Temporal coherence in the perception of tone sequences. Eindhoven, The Netherlands: Institute for Perception Research.

Vos, P. G. (1977). Temporal duration factors in the perception of auditory rhythmic patterns. Scientific Aesthetics, 1, 183-199.

Woodrow, H. (1909). A quantitative study of thythm. Archives of Psychology, 14, 1-66.

Yeston, M. (1976). The stratification of musical rhythm. New Haven, CT: Yale University Press. 